\title{
Multiple timescale spectral analysis
}

\author{
V. Denoël \\ University of Liège, Structural Engineering Division, Chemin des Chevreuils, 1, B52/3, 4000 Liège, Belgium
}

\section{A R T I C L E I N F O}

\section{Article history:}

Received 4 March 2014

Received in revised form

10 December 2014

Accepted 16 December 2014

Available online 19 December 2014

\section{Keywords:}

Perturbation

Background

Resonant

Modal correlation

Cumulants

Volterra series

Evolutionary spectrum

\begin{abstract}
A B S T R A C T
Spectral analysis is a classical tool for the structural analysis of structures subjected to random excitations. The most common application of spectral analysis is the determination of the steady-state second order cumulant of a linear oscillator, under the action of a stationary loading prescribed by means of its power spectral density. There exists however a broad variety of such similar problems, extending the concept to multi degree-of-freedom systems, non Gaussian excitation, slightly nonlinear oscillators or even transient excitations. In this wide class of problems, the cumulants of the response are obtained as the result of the integral of corresponding spectra over the frequency space, which is possibly multidimensional. Application of standard numerical integration techniques may be prohibitive, a reason why the spectral approach is often left aside. Besides, many engineering problems involve a clear timescale separation, usually of those pertaining to the loading and to the mechanical behavior of the system. In these problems, a proper consideration of the timescale separation results in dropping the order of integration by one, at least. This offers the possibility to derive analytical solutions, whenever the order of integration drops to zero, or to make numerical integration competitive. The paper presents this general method, together with some applications in wind and marine engineering.
\end{abstract}

(c) 2014 Elsevier Ltd. All rights reserved.

\section{Introduction}

Many engineering applications are based on the input/output representation of a system, see Fig. 1(a), which is well suited to study the stochastic response of deterministic systems to random input. There is a wide variety of such applications in the various fields of engineering [1-4] among which classical problems of structural and mechanical applications such as the random vibrations of aircraft and spaceships in aerospace engineering, the vibrations of mechanical components [5], the response of civil structures to seismic [6], wind [7] or wave loading [8]. Although the concepts developed in the paper are not limited to the latter domains, the state-of-the-art references are mainly borrowed from structural dynamics, which also serves as the major field of application for the illustrations given in the paper. In many of these applications the timescales $\left\{T^{\star}\right\}$ associated with the system are significantly different from those $\left\{t^{\star}\right\}$ of the loading (input) so that the response turns out to be a composition of components with different timescales. The cases where $\left\{t^{\star}\right\} \ll\left\{T^{\star}\right\}$ or $\left\{T^{\star}\right\} \ll\left\{t^{\star}\right\}$ are the ground motivation for this paper.

Traditional simulation techniques are rather inefficient in solving these kinds of problems as they would have to simultaneously resolve the different scales, which translates in very long simulations with very short timesteps $[9,10]$.

\footnotetext{
E-mail address: v.denoel@ulg.ac.be
}

Alternatively stochastic spectral analysis appears as an appealing tool to solve these problems. Indeed, the loading is usually given with a spectral representation, i.e. a set of spectra defined on multidimensional frequency spaces. Among them, the well-known power spectral density, defined for $\omega \in \mathbb{R}$, represents the distribution of the second cumulant (the variance) over the frequency domain. More generally, the $j$ th-order spectrum represents the distribution of the $j$ th stationary cumulant over a frequency domain $\mathbb{R}^{j-1}$. The objective of a spectral analysis is to determine the spectra of the response in terms of those of the input and eventually integrate them in the corresponding frequency spaces in order to determine the cumulants of the response. Spectral analysis develops in various versions: (i) the Gaussian and the nonGaussian versions, i.e. limited to statistical order 2 or not, e.g. [11-15], (ii) the single degree-of-freedom (SDOF) and the multiple degree-of-freedom (MDOF) versions, the latter being well known for the second order [5,7] and theoretically established for higher order analysis [13,16], (iii) the steady-state response analysis, as in most common applications, but also with evolutionary stochastic analyses, a concept formalized by Priestley [17], but that still receives much interest [18], especially in seismic engineering [19,20] and (iv) although the concept of spectral analysis hinges on the underlying assumptions of linearity and of the superposition principle, both the stochastic linearization [21] (as well as its higher order generalizations [22-24]) and the Volterra series 
a

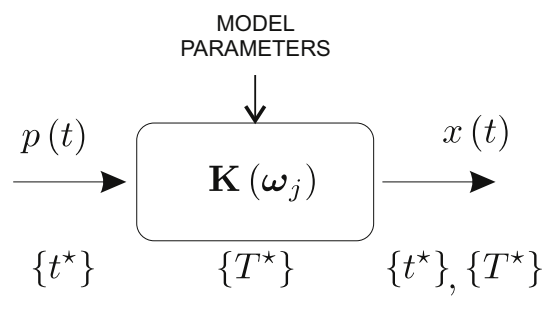

b

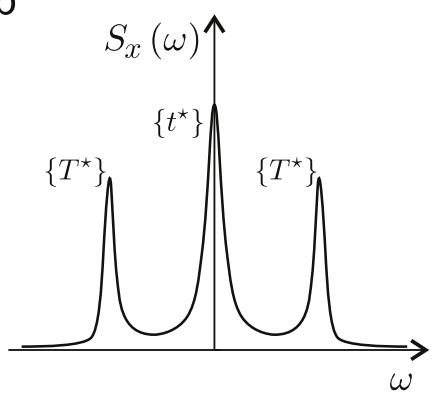

C

$$
B_{x}\left(\omega_{1}, \omega_{2}\right)
$$

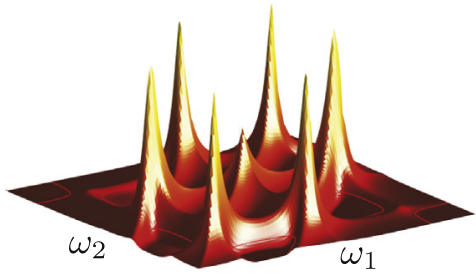

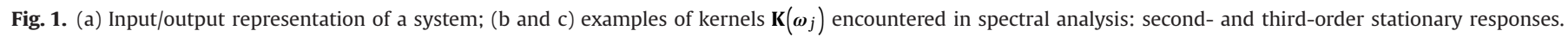

model [25-27] are interesting ways to extend its domain of applicability to systems with mild nonlinearities. In all these versions of the spectral analysis, a canonical form for the $j$ th order spectrum of the response (or contribution thereof in the nonlinear case) is

$\mathbf{S}_{\mathbf{x}}\left(\boldsymbol{\omega}_{j}\right)=\mathbf{K}\left(\boldsymbol{\omega}_{j}\right) \mathbf{S}_{\mathbf{p}}\left(\boldsymbol{\omega}_{j}\right)$

where $\boldsymbol{\omega}_{j}=\left\{\omega_{1}, \ldots, \omega_{j-1}\right\}$ with $j \geq 2$ gathers the independent variables of the frequency space, $\mathbf{K}\left(\boldsymbol{\omega}_{j}\right)$ is a frequency kernel function and $\mathbf{S}_{\mathbf{p}}\left(\boldsymbol{\omega}_{j}\right)$ and $\mathbf{S}_{\mathbf{x}}\left(\boldsymbol{\omega}_{j}\right)$ respectively stand for the $j$ th-order spectra of the loading and of the response. A modified version, adding time $t$ as an additional independent variable, is required in case of evolutionary spectrum [19]. The spectral analysis then consists in the determination of

$\mathbf{k}_{j}=\int \underset{\mathbb{R}^{j-1}}{\cdots} \mathbf{S}_{\mathbf{x}}\left(\omega_{j}\right) d \omega_{j}$

which represents the $j$ th cumulant of the response (or a contribution thereof in the nonlinear case). Examples of such kernels are sketched in Fig. 1(b and c).

The existence of multiple timescales in the response translates into the existence of several well-distinct peaks in the spectra, see Fig. 1(b and c). To handle multiple timescales in the response is thus less problematic than in a crude Monte Carlo simulation. Indeed, the spectral analysis smartly solves the possible issue with an adequate distribution of integration points necessary to determine $\mathbf{k}_{j}$ in (2). Additionally to those used to properly capture the loading $\mathbf{S}_{\mathbf{p}}\left(\boldsymbol{\omega}_{j}\right)$, they have to be distributed in tiny zones around the poles of the kernel $\mathbf{K}\left(\boldsymbol{\omega}_{j}\right)$, i.e. the natural frequencies in case of a linear system.

For $j=2$, some authors avoid the difficulty to justify the truncation of the domain of integration with a suitable change of variable, mapping the infinite frequency domain $\mathbb{R}$ onto a finite interval one [28]. It seems that the concept could be extended to higher dimensional integration $(j>2)$. In other contributions, robust adaptive quadrature schemes [29,30] provide accurate results with limited computational investment, especially if the known location of the resonance peaks is considered to initiate the iterative numerical integration. Although adaptive quadrature is extended to high-dimensional integration [31,32], integration of high-dimensional spectra such as the bispectrum $(j=3)$ and the trispectrum $(j=4)$ has been reported to require excessively large computation times [33,34], mainly because integration in higher dimensions actually requires an exponentially growing number of integration points. Monte Carlo sampling techniques [9] could help accelerating the numerical integration in high-dimensional spaces, but it still requires a tremendous amount of integration points, even if cut by a large factor compared to adaptive quadrature. The end pursued in this paper is to provide, thanks to a timescale separation technique, simplified expressions for these integrals.

Evidences of excessively large computation times trigger the questioning on the applicability of the spectral analysis up to a large order, say fourth order, for large systems. By large systems, we mean those who require a complex finite element modeling with a large number $k$ of DOFs in which case the establishment of the spectral matrix of the loading requires a significant time per integration point $\boldsymbol{\omega}_{j}$. This number is of order $10^{6}$ today. The larger the size $k \times \cdots \times k$ (j times) of that matrix, the worse, of course. Linear systems with a large number of DOFs are analyzed in their modal basis, in which case (1) represents a modal analysis and the size of matrices $\mathbf{S}_{\mathbf{p}}$ and $\mathbf{S}_{\mathbf{x}}$ drops to a few tens or hundreds per dimension. The projection of the spectral representation of the loading (input) in the modal basis, i.e. the determination of $\mathbf{S}_{\mathbf{p}}\left(\boldsymbol{\omega}_{j}\right)$, definitely represents the most important part of the computational burden. Indeed, this operation requires the projection of costly multidimensional nodal matrices. Further observing that this operation has to be repeated for a large number of integration points $\boldsymbol{\omega}_{j}$, this explains why usage of a modal basis reduction only partly solves the computational issue, and thus why only the theoretical setting of the non-Gaussian spectral analysis that was extensively studied in the mid 1990s (e.g. $[11,35,15,36]$ ) is practically limited to structures with very few DOFs ([37]). On the other hand, nonlinear systems with a large number of DOFs are usually analyzed by means of model reduction techniques or statistical linearization concepts, in which case a linear modal analysis follows, which extends the validity of the above observation.

The second-order spectral analysis is now implemented in many structural analysis softwares, be they commercial or not. Large power spectral density matrices of loading are projected in the modal space and the resulting spectra are integrated with accurate numerical methods. Although not completed with a simple click yet, these operations necessitate reasonable computational times, thanks to the resources available on today's personal computers. Even though the number of transistors on a micro-processor chip doubles every 18 months according to Moore's law, acceptable computational times for the third- and the fourth-order analysis of large systems is not to be expected in a near future, at least with a crude application of (2). Looking backward in time however reveals interesting perspectives. In 1961, driven by the need to provide an efficient way of calculating the second- order response of structures subjected to buffeting forces (what is actually rather fast today), Davenport suggested to decompose the response in two components, the background and resonant components [38]. In that approach, the number of integration points thus dropped to the sole natural frequencies of the (possibly very large) structural model. With the benefit of hindsight, we can see this method as the leading order solution of a multiple timescale approach, in which the slow timescale related to the wind loading activates the fast structural dynamic responses 
in an independent timescale. Operationally the method was however presented as a replacement of the actual power spectral density of the modal loading by a constant value, i.e. a white noise. Bouncing ideas off, Preumont has suggested to replace the loading by a piecewise constant approximation which finally results in a semi-analytical approach, thanks to some closed-form integrations [5]. This step was half-way between Davenport's solution and today's practice which requires many integration points. This little piece of history gives a glimpse of the two options for a possible handling of third- and fourth-order analyses of very large structures: either extend Davenport's approach or formally consider a timescale separation in the loading and in the system behavior, either proceed with semi-analytical estimation of the third- or fourth-order integrals. The second option was studied in [31] without a frank success. The first of these two options is considered and developed in this paper.

Multiple scales are a perturbation method that consists in considering that the solution of a problem simultaneously evolves along different dynamics. Actually, the formal mathematical setting of the method perfectly suits initial and boundary value problems, expressed by means of ordinary or partial differential equations. A first application of multiple scales in stochastic dynamics concerns the early attempts at deriving approximate solutions of the Fokker-Planck-Kolmogorov equation [39], for instance when the system memory is significantly different from the input correlation time. Another famous development concerns the WKB approximation of the Schroedinger equation [33]. Starting from there we do not pretend to duplicate the comprehensive reviews of applications of random perturbation methods that already exist in the literature, e.g. [3]. Among others however, in stochastic mechanics, it is worth noting that perturbation methods are mainly used in the presence of small nonlinearities, since [40] the nonlinear response is expressed as a perturbation of the linearized system, see e.g. works initiated by [41]. The perturbation methods are thus usually developed by the extension of deterministic nonlinear mechanics, i.e. as a small perturbation of a linear system.

To the author's knowledge, to invoke a multiple scales technique in a stochastic context, where the response is obtained as the result of an integral in the frequency domain, is a novel contribution. Some exceptions in buffeting wind engineering - where the use of a multiple timescale method was however not coined as such encompass the buffeting response as developed by Davenport [38], the derivation of the approximations for the covariances of modal amplitudes [42] and the derivation of the third-order cumulant of the response of an SDOF linear system subject to lowfrequency turbulence [43]. The multiple timescale spectral analysis is an extension of those examples that offers fast and accurate approximations of statistical moments and cumulants (of any order) in the framework of a spectral analysis. The extension is valid for the wide variety of problems discussed before and is restricted by the sole condition on the timescale separation. We are thus not limited to Markovian processes as in any method based on the Fokker-Planck-Kolmogorov or the moment equations.

\section{Theoretical background}

\subsection{Deterministic systems in the fourier domain}

In a deterministic setting, the MDOF response $\mathbf{x}(t)$ (output) of a passive linear system may be written under the following general form

$\mathbf{x}=\int_{-\infty}^{+\infty} \mathbf{h}(t-\tau) \mathbf{p}(\tau) d \tau$ with $\mathbf{h}(t)$ being the impulse response function of the system and $\mathbf{p}(t)$ the multiple-point excitation (input), see e.g. [44]. The frequency domain response $\mathbf{X}(\omega)$ of the system emerges by the application of the Fourier transform operator

$\mathcal{F}[\mathbf{y}(t) ; \omega]=\int_{-\infty}^{+\infty} \mathbf{y}(t) e^{-\mathrm{i} \omega t} d t$

to each member of (3), with $i$ the complex unit, resulting in

\section{$\mathbf{X}=\mathbf{H P}$}

where $\mathbf{X}(\omega)=\mathcal{F}[\mathbf{x}(t) ; \omega], \mathbf{H}(\omega)=\mathcal{F}[\mathbf{h}(t) ; \omega]$ and $\mathbf{P}(\omega)=\mathcal{F}[\mathbf{p}(t) ; \omega]$, as a consequence of the duality theorem.

Slightly nonlinear systems may be accurately represented with Volterra models $[45,46]$ by the replacement of a nonlinear operator by a series of linear operators, in a fashion that is very similar to Taylor expansion for functions. With this concept, the MDOF response $\mathbf{x}(t)$ of a nonlinear system reads

$\mathbf{X}=\sum_{j=1}^{+\infty} \int \ldots \int \mathbf{h}_{j}\left(t-\tau_{1}, \ldots, t-\tau_{j}\right) \prod_{r=1}^{j} \mathbf{p}\left(\tau_{r}\right) d \tau_{1} \ldots d \tau_{j}$

with $\mathbf{h}_{j}\left(t_{1}, \ldots, t_{j}\right)$ being the $j$ th-order Volterra kernel (of the MDOF problem). In the frequency domain, the response of a Volterra system is also fully characterized by its higher order frequency response functions, which are defined by a multiple-fold Fourier transform of the kernels, i.e.

$\mathbf{H}_{j}\left(\boldsymbol{\omega}_{j}\right)=\int \underset{\mathbb{R}^{j}}{\ldots} \int e^{-\mathrm{i}(\omega 1 \tau 1+\cdots+\omega j \tau j)} \mathbf{h}_{j}\left(\tau_{1}, \ldots, \tau_{j}\right) d \tau_{1} \ldots d \tau_{j}$.

\subsection{Spectral analysis}

The frequency domain approach is particularly well suited to study the response of deterministic structures subjected to stochastic loading, since both the response $\mathbf{X}(\omega)$ and the loading $\mathbf{P}(\omega)$ are viewed as stochastic processes. Under rather general conditions [7], the spectral density of the response $\mathbf{S}_{\mathbf{x}}(\omega)$, defined as a mean square expectation, is expressed as a function of the spectral density of the loading $\mathbf{S}_{\mathbf{p}}(\omega)$ as

$$
\mathbf{S}_{\mathbf{x}}=\mathbf{H} \mathbf{S}_{\mathbf{P}} \mathbf{H}^{*}
$$

or

$S_{x_{i 1 i 2}}=\sum_{j_{1}} \sum_{j_{2}} H_{i 1 j 1} S_{p_{j 1} j_{2}} H_{j_{2} i_{2}}^{*}$

where symbol * represents the complex conjugate. This secondorder response $(j=2)$ is well a particular case of (2) with $\boldsymbol{\omega}_{j} \equiv \omega$ and $\mathbf{K}(\omega) \equiv \mathbf{H}(\omega) \mathbf{H}^{*}(\omega)$.

Higher order spectra, such as the bispectrum $B_{x_{11} i_{2 i}}\left(\omega_{1}, \omega_{2}\right)$ and the trispectrum $T_{x_{i 12 i 3 i 4}}\left(\omega_{1}, \omega_{2}, \omega_{3}\right)$ related to the higher order cross-cumulants of the structural response find applications in various fields of science and engineering [47]; they are also expressed in terms of the bispectrum and trispectrum of the loading [16]. In an MDOF context to be general, and under stationary conditions, the third- and fourth-order spectra of the response read

$$
\begin{aligned}
B_{x_{11 i 2 i 3}}\left(\omega_{1}, \omega_{2}\right)= & \sum_{\left\{j_{1}, j 2, j 3\right\}} H_{i 1 j 1}\left(\omega_{1}\right) H_{i 2 j 2}\left(\omega_{2}\right) H_{i 3 j 3}^{*}\left(\omega_{1}+\omega_{2}\right) \\
& B_{p_{j 1 j 2 j 3}}\left(\omega_{1}, \omega_{2}\right)
\end{aligned}
$$


and

$$
\begin{aligned}
& T_{x i 1 i 2 i 3 i 4}\left(\omega_{1}, \omega_{2}, \omega_{3}\right) \\
& =\sum_{\left\{j_{1}, j_{2}, j_{3}, j_{4}\right\}} H_{i 1 j_{1}}\left(\omega_{1}\right) H_{i 2 j 2}\left(\omega_{2}\right) H_{i 3 j 3}\left(\omega_{3}\right) H_{i 4 j 4}^{*}\left(\omega_{1}+\omega_{2}+\omega_{3}\right) \\
& \quad B_{p_{j_{1} j_{2} j_{3} j_{4}}\left(\omega_{1}, \omega_{2}, \omega_{3}\right) .}
\end{aligned}
$$

Again, they may be viewed as particular cases of (2). Similar expressions are also developed for nonlinear and evolutionary systems [17], which finally makes (2) very general.

\subsection{Timescale separation}

The backdrop of this paper is related to problems where the intrinsic timescales of the loading $\mathbf{S}_{\mathbf{p}}\left(\boldsymbol{\omega}_{j}\right)$ are different from those of the system $\mathbf{K}\left(\boldsymbol{\omega}_{j}\right)$. While the latter ones are generally attributed to the poles in the kernel function $\mathbf{K}\left(\boldsymbol{\omega}_{j}\right)$, those pertaining to the loading require a more formal definition.

Invoking linearity, the intrinsic structural timescales of a linear MDOF system are those of its natural modes of vibration. Thanks to stochastic linearization and Volterra models, this definition is straightforwardly extended to mild nonlinear models. Analysis in a modal basis further simplifies the idea, as only some mode shapes remain in the description of the structural displacement filed. There are as many timescales as the number of considered structural mode shapes, with a restriction on counting their multiplicity. They are defined as the natural periods

$T_{i}^{\star}=\frac{2 \pi}{\bar{\omega}_{i}}$

also expressed in terms of natural circular frequencies $\bar{\omega}_{i}$. Associated with each mode of vibration is another characteristic time known as memory which is of order $T_{i}^{\star} \mid \xi_{i}$ where $\xi_{i}$ is the damping coefficient in mode $i$. Although memory could be seen as a very slow timescale, on account that damping ratios are very small in typical applications, a frequency domain approach reveals that it is rather associated with the period of beating around the natural frequency of the oscillator or, in other words, with the width of the peak in the frequency response function [5]. To this slow timescale is usually attributed very few importance as it does not play a central role in the second-order response. However, interesting interactions between this slow timescale and the slow timescale of the loading appear for statistical orders equal to 3 or more [43], a reason why it requires a dedicated attention in this general framework. Notice finally that some of the timescales $T_{i}^{\star}$ related to the system might be equal. This is not an issue.

At order $j \geq 2$, we define the timescale $t_{j}^{\star}$ of a stationary loading by

$\frac{2 \pi}{t_{j}^{\star}}:=\alpha_{j}=\left(\frac{\int \cdots \int_{\mathbb{R}^{j-1}}\left\|\boldsymbol{\omega}_{j}\right\|_{2}^{2} \mathbf{S}_{\mathbf{p}}\left(\boldsymbol{\omega}_{j}\right) d \boldsymbol{\omega}_{j}}{\int \cdots \int_{\mathbb{R}^{j-1}} \mathbf{S}_{\mathbf{p}}\left(\boldsymbol{\omega}_{j}\right) d \boldsymbol{\omega}_{j}}\right)^{1 / 2}$

where $\alpha_{j}$ is a characteristic frequency of the loading at order $j$ and where $\left\|\Omega_{j}\right\|_{2}$ represents the 2-norm of the frequency vector. At order $j=2$, this definition is particularly well suited to narrowband processes - such as wave height, e.g. [48] - in which case $\alpha_{2}$ provides the center frequency of the band, or to processes with a decreasing frequency content such as wind turbulence, in which case $\alpha_{2}$ is similar to the center of mass of the one-sided distribution. Although different from $\alpha_{2}$, the higher order timescales $(j \geq 3)$ are fundamentally of the same order of magnitude as $\alpha_{2}$. This results from the definition of the stationary spectra as a mathematical expectation of a product of $j$ factors; in the definition (13) the orders of magnitude related to $\mathbf{X}$ therefore simplify, which finally yields $O\left(\alpha_{j}\right)=O\left(\alpha_{2}\right), \forall j \geq 3$.

Nonstationary loadings such as earthquakes come with another timescale $t_{1}^{\star}$ which is usually long compared to the other timescales involved in the problem. In this paper, we restrict the development to the cases of practical interest, where $t_{1}^{\star}$ is one or several orders of magnitude larger than $t_{j}^{\star}(j \geq 2)$ and $T_{i}^{\star}$.

Timescale separation means that the $t_{j}^{\star}$ are one or several orders of magnitude larger or smaller than the timescales $T_{i}^{\star}$ of the system. Formally, the ratios

$\varepsilon_{i j}=\frac{T_{i}^{\star}}{t_{j}^{\star}}$

are thus either small $\left(\varepsilon_{i j} \ll 1\right)$ or large $\left(\varepsilon_{i j}^{-1} \ll 1\right)$. In all other cases, there is no other option than to recourse to the heavy numerical integration of the spectra as in (2).

\subsection{Integrals with small parameters}

Small parameters are the keypoint in perturbation methods. The problem under consideration involves two sets of small parameters: the structural damping coefficients $\xi_{i}$ and the timescale separation ratios, i.e. either $\varepsilon_{i j}$ or $\varepsilon_{i j}^{-1}$. They are responsible for the sharpness of the peaks in the spectra and for the distinctness of both groups of peaks, respectively.

There is a wide variety of perturbation methods related to differential equations. Matched or patched asymptotic methods, strained coordinates, multiple scales [49] or the WKB (WentzelKramers-Brillouin) approximation [50-52] are just some of the most common ones. It would thus be tempting to transform the problem under consideration under the format of a differential equation. In theory, this format is obtained by a multidimensional Fourier transform of (1), which yields the well-known Lyapunov equation for the correlation matrix in the case $j=2$ and its higher order extensions for $j \geq 3$. This differential equation format in the time domain is well suited to the application of perturbation methods. However it would be drastically inefficient in the current context, since the Fourier transform of the spectra of various orders $\mathbf{S}_{p}\left(\boldsymbol{\Omega}_{j}\right)$ would require numerical estimations (before multidimensional inverse Fourier transform), for a huge number of frequencies, precisely what we seek to avoid.

The problem thus requires being considered in its integral form (2). A typical way to handle integrals with small parameters hinges on Watson's lemma [53] or the steepest descent or the saddle point method [53] and requires a series expansion of the integrand. Practically their applicability is however limited to problems with local contributions only, i.e. resulting from small regions of the domain of integration, in contrast with global (or non-local) contributions which result from contributions distributed over the whole range of integration. Because there exists such a non-local contribution in the problem at hand - which is an indicator of the simultaneous existence of different timescales -, it is required to consider them as such. Existing methods are then rather scarce in the presence of both local and non-local contributions. There is however one method proposed by Hinch that seems to be promising in view of its extension to the considered problem. It is instructive to illustrate this method with the example taken from [53] (p. 38).

Consider the integral of the function $f(x)=(\epsilon+x)^{-1 / 2}$ with $\epsilon \ll 1$ over the domain $x \in[0 ; 1]$, which is depicted in Fig. 2(a). There exists a local contribution to this integral, coming from the domain $x \in[0 ; \epsilon]$, in which the integrand is of order $\epsilon^{-1 / 2}$. Multiplying the extent of the region and the order of magnitude of the function provides the order of magnitude of that local contribution, namely $\epsilon^{1 / 2}$. Besides, there exists also a global contribution to 

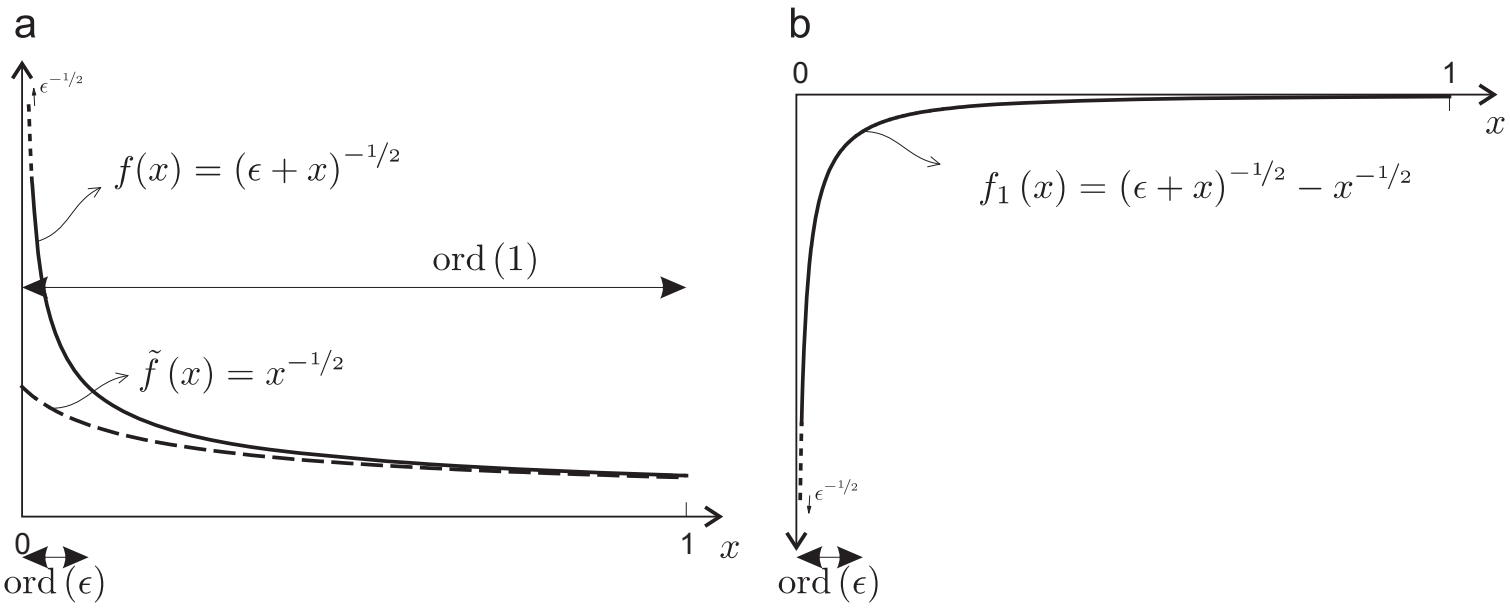

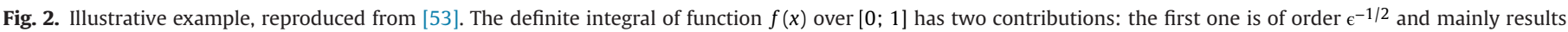

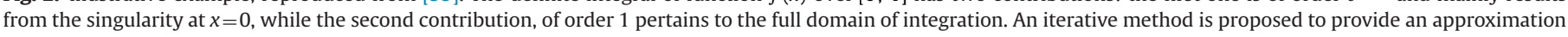
of the exact result, for small $\epsilon$.

the integral, which is a result on the whole domain of integration $x \in[0 ; 1]$. In that case the function is of order 1 and it is readily seen that the global contribution is of order 1 . Consequently the leading order contribution to the integral is the global one, and it may be captured by replacing the function $f$ by the approximation $\tilde{f}(x)=x^{-1 / 2}$, which is valid in the whole domain except in the tiny zone $x \in[0 ; \epsilon]$, see Fig. 2(a). The integral of the first approximation $\tilde{f}$ over the range $[0 ; 1]$ is equal to 2 , i.e. of order 1 as expected. The next order correction to the integral may be obtained by subtracting off the first approximation $\tilde{f}$ from the original function, which yields with no approximation

$\int_{0}^{1}(\epsilon+x)^{-1 / 2} d x=2+\int_{0}^{1}\left((\epsilon+x)^{-1 / 2}-x^{-1 / 2}\right) d x$

where the residual $f_{1}=f-\tilde{f}=(\epsilon+x)^{-1 / 2}-x^{-1 / 2}$, see Fig. 2(b), then requires being integrated. Reassessment of the local/global contributions in the same way indicates now a dominance of the local contribution. Introduction of a stretched coordinate $x=\epsilon u$ resets the contribution interval to a unit measure $u=\operatorname{ord}(1)$ so that the residual function reads $\epsilon^{-1 / 2}\left((1+u)^{-1 / 2}-u^{-1 / 2}\right)$ and the remaining integral estimated along $u \in\left[0 ; \epsilon^{-1}\right]$ or $u \in[0 ;+\infty]$ has the same leading order contribution. The definite integral in (15) thus amounts to $-2 \epsilon^{1 / 2}$, i.e. a local contribution of order $\epsilon^{1 / 2}$, as expected. Finally, the sum of the two contributions that were identified, i.e. $2-2 \epsilon^{1 / 2}$, corresponds to the leading order terms of the series expansion of the exact solution which is actually given by $2\left((1+\epsilon)^{1 / 2}-\epsilon^{1 / 2}\right)$.

\section{Multiple timescale spectral analysis}

\subsection{Generalities}

The analytical method presented by Hinch is now replicated in the context of the problem at hand, i.e. the determination of the integral of $\mathbf{S}_{\mathbf{x}}\left(\boldsymbol{\omega}_{j}\right)=\mathbf{K}\left(\boldsymbol{\omega}_{j}\right) \mathbf{S}_{\mathbf{p}}\left(\boldsymbol{\omega}_{j}\right)$, with the difference however that the function to be integrated is not completely given explicitly. Indeed, on one hand, it results from a presumably complex numerical model for the computation of the loading spectrum $\mathbf{S}_{\mathbf{p}}\left(\boldsymbol{\omega}_{j}\right)$, which is to be estimated for a number of integration points $\omega_{j}$ that is as limited as possible. On the other hand, it is however assumed that the kernel function $\mathbf{K}\left(\boldsymbol{\omega}_{j}\right)$ is known in a closed form, usually a combination of rational fractions, as it results from simple linear (or linearized) structural dynamics.

The first step of the method consists in recognizing the existence of different timescales, i.e. different peaks in $\mathbf{S}_{\mathbf{x}}\left(\boldsymbol{\omega}_{j}\right)$ which are thus sufficiently distinct, in a multiple timescale spirit. This operation identifies the different contributions to the integral. Sharp peaks are local contributions to the integral while wideband spectral regions result in global contributions. Because of the timescale separation, significant contributions to the integral might only result from the regions in the frequency space where either one of these two factors is maximum. To have either one of the both factors maximum is however not a sufficient condition for the significance of that contribution, since other peaks might get the edge. All possible combinations should thus be analyzed and sorted out, specifically for each and every new problem. However depending on their nature, the peaks might be classified into three categories, depending on the factor they maximize, $\mathbf{S}_{\mathbf{p}}\left(\boldsymbol{\omega}_{j}\right), \mathbf{K}\left(\boldsymbol{\omega}_{j}\right)$ or some of the factors in $\mathbf{S}_{\mathbf{p}}\left(\boldsymbol{\omega}_{j}\right)$ and $\mathbf{K}\left(\boldsymbol{\omega}_{j}\right)$ provided they are both expressed as products themselves. These cases are successively detailed next.

\subsection{The background component}

The background component corresponds to the peak(s) in the loading spectrum $\mathbf{S}_{\mathbf{p}}\left(\boldsymbol{\omega}_{j}\right)$. There might be several local peaks or crests in the loading spectrum, but at this stage, we only focus on the regions of the frequency space with the most important contribution, i.e. typically in or on a hypersphere with radius $\alpha$. Depending on the relative smallness of the characteristic frequencies of the loading $\alpha_{j}$ and those of the structure $\bar{\omega}_{i}$, the local behavior of the kernel $\mathbf{K}\left(\boldsymbol{\omega}_{j}\right)$ differs.

First, let us consider the case where $\alpha_{j} \ll \bar{\omega}_{i}(\forall i, \forall j)$. The local behavior of the kernel $\mathbf{K}\left(\boldsymbol{\omega}_{j}\right)$ in the vicinity of the origin might usually be established without much trouble; we thus presume a local approximation $\tilde{\mathbf{K}}$ is available. For instance, it is constant and corresponds to the well-known quasi-static solution in the case of a linear oscillator. In the other case where $\alpha_{j} \gg \bar{\omega}_{i}$, another asymptotic behavior is generally available, for instance the wellknown inertial regime solution in the case of a linear oscillator. Another approximation $\tilde{\mathbf{K}}$ is thus also assumed to be available, in the high frequency range. In both cases, the approximation $\tilde{\mathbf{K}}$ needs to be such that

$\int \cdots \int_{\mathbb{R}^{j-1}} \tilde{\mathbf{K}}\left(\boldsymbol{\omega}_{j}\right) \mathbf{S}_{\mathbf{p}}\left(\boldsymbol{\omega}_{j}\right) d \boldsymbol{\omega}_{j}$ 
exists and takes on a simpler analytical form than the original problem (2).

Usually approximations of the form

$\tilde{\mathbf{K}}\left(\boldsymbol{\omega}_{j}\right)=\mathbf{K}_{0} \prod_{i=1}^{j} \omega_{i}^{p_{i}} k_{i}^{-q_{i}}$

are suitable for the purpose of the current needs. In structural dynamics problems, $k_{i}$ represents a generalized stiffness, $i \in[1 ; j]$, and $q_{i}$ are integer powers, while $\mathbf{K}_{0}$ is some vectorial function of order 1 . The factors $\omega_{i}^{p_{i}}$ in (17) appear in the asymptotic expansion of $\mathbf{K}\left(\boldsymbol{\Omega}_{j}\right)$ in the high-frequency asymptotes (inertial regimes), see Problem 5 below, or even when the kernel corresponds to velocities, see Problem 2 below.

The case of the second-order linear oscillator $(j=1)$ is recovered from this general formulation, by setting $q_{1}=2$ and either $p_{1}=0$ in the case $\alpha \ll \bar{\omega}$, either $p_{1}=-4$ in the case $\alpha \gg \bar{\omega}$. Substitution of (17) into (16) yields the background component

$\mathbf{k}_{j, b}=\mathbf{K}_{0}\left(\prod_{i=1}^{j} k_{i}^{-q_{i}}\right) \int_{\mathbb{R}^{j-1}} \ldots \int_{i=1} \prod_{i}^{j} \omega_{i}^{p_{i}} \mathbf{S}_{\mathbf{p}}\left(\boldsymbol{\omega}_{j}\right) d \boldsymbol{\omega}_{j}$

where the remaining integral is expected to exist, otherwise another appropriate local approximation has to be sought. All the more, based on the analytical information on the loading, it is usually possible to express this integral explicitly, e.g. as a function of the variance of the loading, so that finally the background component of the response does not require any numerical integration anymore and

$\mathbf{k}_{j, b}=\mathbf{K}_{0}\left(\prod_{i=1}^{j} k_{i}^{-q_{i}}\right) \mathbf{k}_{j, p}$

where $\mathbf{k}_{j, p}$ precisely represents the integral in (18), i.e. some statistical moment of the loading.

\subsection{The resonant and mixed background/resonant components}

Subtracting off the background approximation $\tilde{\mathbf{K}}\left(\boldsymbol{\omega}_{j}\right) \mathbf{S}_{\mathbf{p}}\left(\boldsymbol{\omega}_{j}\right)$ from the initial problem, we are left with the approximation of

$\int \underset{\mathbb{R}^{j-1}}{\cdots}\left[\mathbf{K}\left(\boldsymbol{\omega}_{j}\right)-\tilde{\mathbf{K}}\left(\boldsymbol{\omega}_{j}\right)\right] \mathbf{S}_{\mathbf{p}}\left(\boldsymbol{\omega}_{j}\right) d \boldsymbol{\omega}_{j}$

which does not feature any significant contribution in the background domain anymore, in or on the hypersphere where $\boldsymbol{\omega}_{j} \lesssim \boldsymbol{\alpha}$. Resonant and mixed background/resonant components are found to be the remaining ones in this residual. The analysis of the different contributions to this integral has to be performed specifically for each problem. Following the same outline as for the example before, we may extrapolate that the most important contributions are attributable to the regions of the frequency space where the poles of $\mathbf{K}\left(\boldsymbol{\omega}_{j}\right)$ are located; they correspond to resonant components. In those regions, the loading spectrum $\mathbf{S}_{\mathbf{p}}\left(\boldsymbol{\omega}_{j}\right)$ might be very small which thus results in a small resonant component. Depending on the original expression of the kernel $\mathbf{K}\left(\boldsymbol{\omega}_{j}\right)$ and the loading spectrum $\mathbf{S}_{\mathbf{p}}\left(\boldsymbol{\omega}_{j}\right)$, other regions of the domain may contribute more significantly. They correspond to smaller values of the factor in the square brackets, but much larger values of the loading spectrum. These components are classified as mixed background/ resonant components. Some examples are given in Section 4. There is a priori no means to rank the relative importance of these terms, not more than with the background one anyway, in a general fashion. It is thus recommended to carefully identify and study the different possible contributions. The following steps have to be repeated sequentially for each identified contribution.

Let us assume that a contribution to the integral in (20) has been identified in the neighborhood of $\boldsymbol{\omega}_{j}=\boldsymbol{\Omega}_{j}$ and extends in the frequency space over a more or less short bandwidth $\boldsymbol{\Delta}_{j}$. Notice the contribution is said to be a peak if all components of $\boldsymbol{\Delta}_{j}$ have the same order of magnitude, a crest otherwise. A new set of coordinates $\boldsymbol{\eta}_{j}$ is then introduced in order to reset the focus of the analysis onto that contribution only

$\boldsymbol{\omega}_{j}=\boldsymbol{\Omega}_{j}+\boldsymbol{\Delta}_{j} \boldsymbol{\eta}_{j}$

The remainder $f_{1}\left(\boldsymbol{\omega}_{j}\right)=\left[\mathbf{K}\left(\boldsymbol{\omega}_{j}\right)-\tilde{\mathbf{K}}\left(\boldsymbol{\omega}_{j}\right)\right] \mathbf{S}_{\mathbf{p}}\left(\boldsymbol{\omega}_{j}\right)$ thus reads

$f_{1}\left[\boldsymbol{\omega}_{j}\left(\boldsymbol{\eta}_{j}\right)\right] d \boldsymbol{\omega}_{j}=\mathcal{K}\left(\boldsymbol{\eta}_{j}\right) \mathbf{S}_{\mathbf{p}}\left[\boldsymbol{\omega}_{j}\left(\boldsymbol{\eta}_{j}\right)\right]\left|\mathbf{J}_{\eta \omega}\right| d \boldsymbol{\eta}_{j}$

where $\mathbf{J}_{\eta \omega}$ is the Jacobian of the transformation and $\mathcal{K}\left(\boldsymbol{\eta}_{j}\right)=\mathbf{K}\left(\boldsymbol{\omega}_{j}\left(\boldsymbol{\eta}_{j}\right)\right)-\tilde{\mathbf{K}}\left(\boldsymbol{\omega}_{j}\left(\boldsymbol{\eta}_{j}\right)\right)$. There is no approximation at this stage of the developments, so that the expression of $\mathcal{K}$ might be somehow cumbersome even in simple applications. The cornerstone of the method is now to derive a local approximation of the product $\mathcal{K}\left(\boldsymbol{\eta}_{j}\right) \mathbf{S}_{\mathbf{p}}\left[\boldsymbol{\omega}_{j}\left(\boldsymbol{\eta}_{j}\right)\right]$ that is bounded, integrable and provides the most important part of its contribution to the integral in the hypersphere of radius $\boldsymbol{\eta}_{j} \simeq \mathbf{1}$. Several methods are provided in the illustrations of Section 4 to obtain simple and accurate approximations. They might be summarized as follows. The use of Laurent series and Padé approximants is usually suitable for $\mathcal{K}$, although some physical intuition or manual simplification on account of the different orders of magnitude might also provide satisfactory results. On the other side, $\boldsymbol{S}_{\mathbf{p}}\left[\boldsymbol{\omega}_{j}\left(\boldsymbol{\eta}_{j}\right)\right]$ might usually be considered as constant across a resonance peak or perpendicularly to a crest, as a result of the timescale separation. In highdimensional and nonlinear problems, it is however seldom that this factor might be considered as a constant in all directions of the frequency space [43,54]. This assumption is valid in at least one direction of the frequency space for resonant terms, and more than one for mixed background/resonant contributions. This statement is better illustrated with examples, see Section 4.

Denoting the new local approximations in the stretched coordinate system with tildes, the resonant (or mixed) contribution associated with the current region of the frequency space reads

$\mathbf{k}_{j, r}=\int \underset{\mathbb{R}^{j-1}}{\ldots} \tilde{\mathcal{K}}\left(\boldsymbol{\eta}_{j}\right) \tilde{\boldsymbol{S}}_{\mathbf{p}}\left[\boldsymbol{\omega}_{j}\left(\boldsymbol{\eta}_{j}\right)\right]\left|\mathbf{J}_{\eta \omega}\right| d \boldsymbol{\eta}_{j}$

where $\tilde{\mathbf{S}}_{\mathbf{p}}\left[\boldsymbol{\omega}_{j}\left(\boldsymbol{\eta}_{j}\right)\right]$ does not depend on all components of $\boldsymbol{\eta}_{j}$ for the reasons given above. Integration along at least one direction might thus be performed explicitly, which thus drops by one the dimensionality of the integral.

Other contributions to the integral are obtained by subtracting off the approximation $\tilde{\mathcal{K}}\left(\boldsymbol{\eta}_{j}\right) \tilde{\mathbf{S}}_{\mathbf{p}}\left[\boldsymbol{\omega}_{j}\left(\boldsymbol{\eta}_{j}\right)\right]$ from the former residual and proceeding with a similar technique. Notice however that the successive subtractions of the local approximations to each peak or crest provide more and more complex residuals. Provided the local approximation covers a region that is limited to the hypersphere of radius $\mathbf{1}$ in the stretched coordinate system, and provided the various contributions are well distinct, it is possible to keep the first residual $f_{1}$ unchanged while iterating, as the subtraction of a local approximation should not affect the local behavior in the neighborhood of other contributions.

\section{Illustrations}

The multiple timescale spectral analysis is illustrated in this section with typical structural engineering problems. The first problem concerns the estimation of the variance of the structural 
response of an SDOF oscillator subjected to a low turbulence buffeting load. The second problem discusses the estimation of the velocity response of an SDOF oscillator. The third problem concerns the estimation of the covariance of modal coordinates and is thus aimed at illustrating the application of the method to MDOF systems. A fourth example illustrates the applicability to higher order moments, such as the third-order cumulant of the response of a SDOF system. Finally, a fifth problem related to the surge response of an SDOF offshore structure is considered. Its objective is to illustrate both the applicability to a nonlinear system and the possibility to handle cases where the loading corresponds to the fast dynamics, i.e. the structure responds in its inertial regime in the background response.

To ease comparison with the theoretical developments of Section 3, the same notation is used in different contexts. Unless otherwise stated, $\mathbf{K}$ and $S_{p}$ have different meanings in each problem.

\subsection{Problem 1: variance of the response of an SDOF system}

The stationary variance of the response of a simple oscillator subjected to a stationary excitation is obtained by

$k_{2}=\int_{-\infty}^{\infty} S_{p}(\omega)|H(\omega)|^{2} d \omega$

where $S_{p}(\omega)$ stands for the power spectral density of a lowfrequency forcing process $\left(\alpha \ll \bar{\omega}, T^{\star} \ll t^{\star}\right)$. This integral takes on the canonical form (2) where $\omega_{j}=\omega$ (we thus drop the subscript for simplicity) and
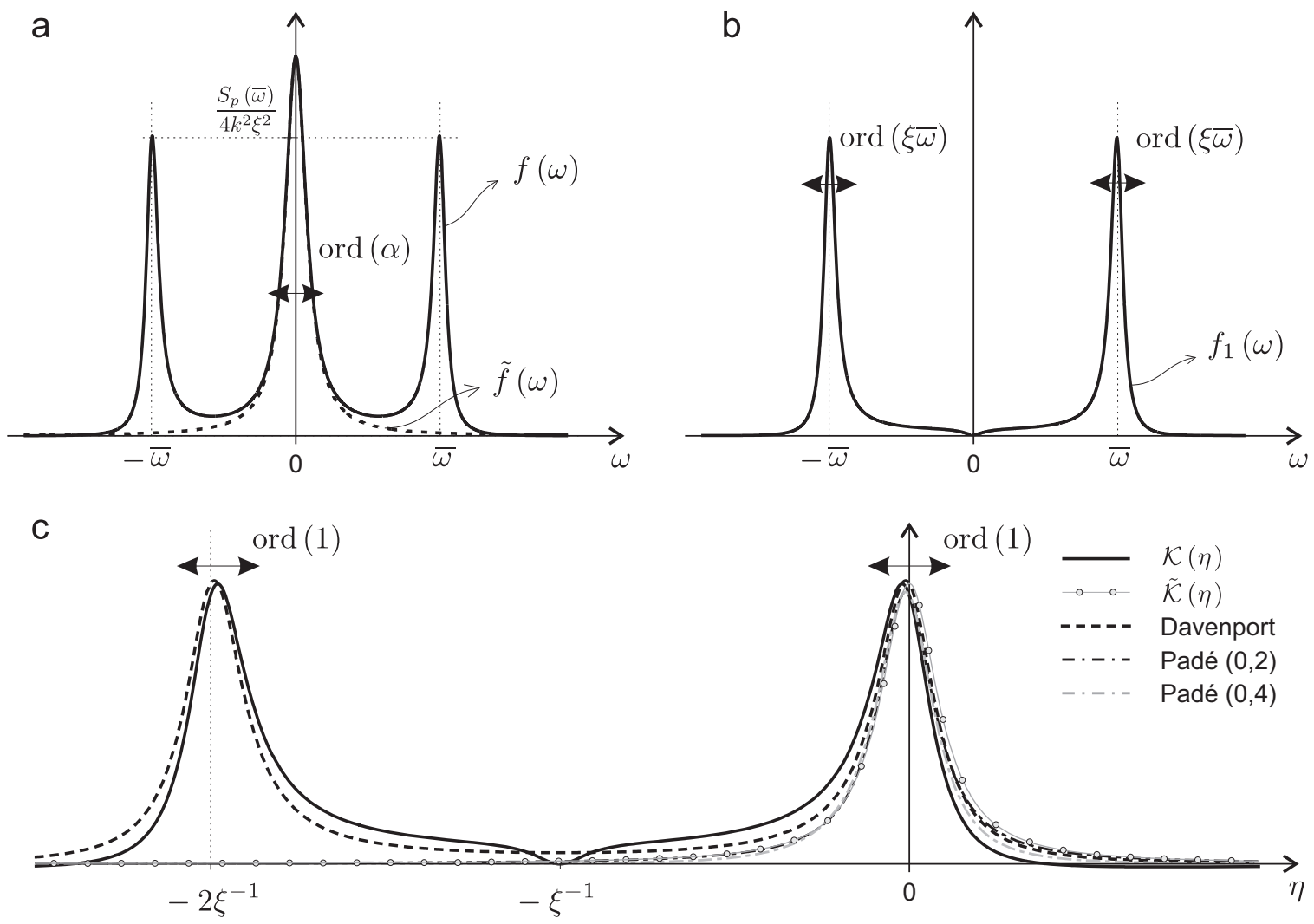

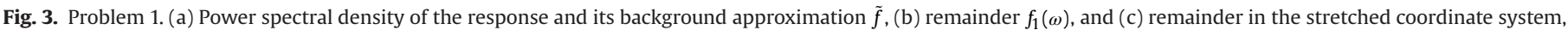
focus on the right peak. The other peak is repelled towards $-\infty$ and a local approximation is derived.

$\mathbf{K}\left(\boldsymbol{\omega}_{j}\right) \equiv|H(\omega)|^{2}=\frac{1}{k^{2}} \frac{1}{\left(1-\left(\frac{\omega}{\bar{\omega}}\right)^{2}\right)^{2}+\left(2 \xi \frac{\omega}{\bar{\omega}}\right)^{2}}$

with $k$ being the stiffness, $\xi$ the damping ratio and $\bar{\omega}$ the circular natural frequency. The computation of this integral arises not only in case of SDOF systems, but also in the analysis of linear multiDOF systems, in their modal basis. This justifies the central place occupied by this integral and the need for a fast an accurate estimation. Beside a simple validation and illustration of the proposed method, this review of a well-known problem also aims at demonstrating that the derivation of an approximate solution is not unique.

In the low-frequency region, the kernel $\mathbf{K}$ is locally approximated by its asymptotic behavior, i.e. $\tilde{\mathbf{K}}=1 / k^{2}$, so that the local approximation of the integrand in the low-frequency region is $\tilde{f}(\omega)=\left(1 / k^{2}\right) S_{p}(\omega)$, see Fig. 3(a), and the background component of the response reads

$k_{2, b}=\frac{1}{k^{2}} \int_{-\infty}^{+\infty} S_{p}(\omega) d \omega=\frac{k_{2, p}}{k^{2}}$

where $k_{2, p}$ represents the second cumulant (the variance) of the loading. Subtracting off the approximation $\tilde{f}(\omega)$ from the initial power spectrum of response provides a first residual

$f_{1}(\omega)=S_{p}(\omega)(\mathbf{K}(\omega)-\tilde{\mathbf{K}})$

that has obviously no more component in the vicinity of the origin, see Fig. 3(b). A formal integration of this residual $f_{1}$ on $\mathbb{R}$ would provide the supplement to the background response $k_{2, b}$ in order to recover the exact variance, with no approximation. It would be tempting to replace $S_{p}(\omega)$ by $S_{p}(\bar{\omega})$ right now, but this would result

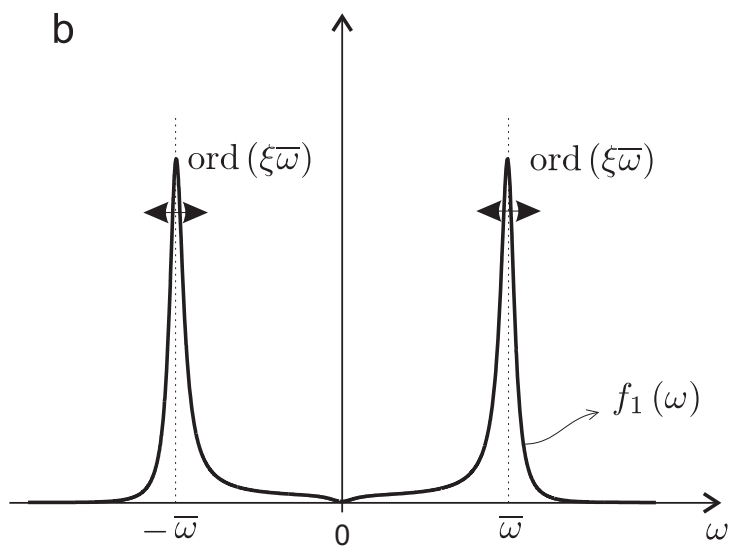


in an approximation of $f_{1}(\omega)$ that is not integrable in the far field, as $\lim _{\omega \rightarrow \pm \infty}(\mathbf{K}(\omega)-\tilde{\mathbf{K}})=-1 / k^{2}$. What makes $f_{1}(\omega)$ integrable in the far field is precisely the decrease of $S_{p}(\omega)$ at high frequency. The only chance to obtain a finite integral is to provide a bounded approximation for $f_{1}$, which translates ipso facto in the need to provide a bounded approximation for $(\mathbf{K}-\tilde{\mathbf{K}})$, should one desire to replace $S_{p}(\omega)$ by $S_{p}(\bar{\omega})$. With the multiple timescale spectral analysis, this issue is solved by providing an approximation for $(\mathbf{K}-\tilde{\mathbf{K}})$, that decreases to zero fast enough at high frequency.

The local/global analysis of the contributions to the integral of the first residual $f_{1}$ indicates that the integral of $f_{1}$ is now mainly composed of two contributions of equal magnitude originating from the two peaks symmetrically laid, centered on $\omega= \pm \bar{\omega}$ and whose extents are of order $\xi \bar{\omega}$, see Fig. 3(b). These contributions are thus of order $S_{p}(\bar{\omega})\left(1 / 4 k^{2} \xi^{2}\right) \xi \bar{\omega}$. The general method requires to sequentially focus on each of them. The contribution related to the peak centered on $\omega=\bar{\omega}$ is studied first and we formalize this close-up view of the problem by introducing the strained coordinate

$\omega=\bar{\omega}(1+\xi \eta)$

with the Jacobian $d \omega / d \eta=\xi \bar{\omega}$. Substitution of (28) into (27) yields

$f_{1}[\omega(\eta)] d \omega=S_{p}[\bar{\omega}(1+\xi \eta)] \mathcal{K}(\eta) \xi \bar{\omega} d \eta$,

with $\mathcal{K}(\omega)$ defined by

$$
\begin{aligned}
\mathcal{K}(\eta) & =|H(\omega(\eta))|^{2}-\frac{1}{k^{2}} \\
& =\frac{1}{k^{2} \xi^{2}} \frac{(1+\xi \eta)^{2}\left(1-2 \xi \eta-\left(4+\eta^{2}\right) \xi^{2}\right)}{4\left(1+\eta^{2}\right)+4 \xi \eta\left(2+\eta^{2}\right)+\xi^{2} \eta^{2}\left(4+\eta^{2}\right)},
\end{aligned}
$$

so that $f_{1}$ is well of order $\xi^{-1}$ along the domain on $\eta=\operatorname{ord}(1)$. This indicates that the stretching has well initiated the reset of the expected contribution to a domain of order 1 . However, as might be seen from Fig. 3(c), the function still exhibits a non-zero asymptote at infinity as well as two peaks; one is located at $\eta=0$ while the other is repelled at $\eta=-2 \xi^{-1}$. From this stage onwards, we thus need to derive a local approximation $\tilde{f}_{1}$ that fits this expression of $f_{1}$ for values of the strained coordinate $\eta=\operatorname{ord}(1)$ at most, as it is desired to focus on one peak at a time, and that decreases fast enough in the far field ( $\eta \gg 1)$, in order to ensure the convergence of the integral and prohibit interaction with other contributions. There exists several local approximations that satisfy these conditions. Some examples are given next.

Because the analytical expression of $\mathcal{K}(\eta)$ is not too heavy, a first local approximation is obtained manually from (30), assuming $\eta=\operatorname{ord}(1)$ and $\xi \ll 1$, e.g. discarding terms as $\xi \eta$ in front of 1 . This results in the first approximation

$\tilde{\mathcal{K}}(\eta)=\frac{1}{k^{2} \xi^{2}} \frac{1}{4\left(1+\eta^{2}\right)}$.

Notice that the degree of the denominator has dropped from 4 to 2 , i.e. $\mathcal{K}$ dropped from two poles to one only, which demonstrates well the reduction to a single peak in the vicinity of $\eta=0$, i.e. $\omega=+\bar{\omega}$. Notice also $\lim _{\eta \rightarrow \pm \infty} \tilde{\mathcal{K}}=0$ and $\left|\int_{\mathrm{R}} \tilde{\mathcal{K}} d \eta\right|<\infty$. Because now $\tilde{\mathcal{K}}$ is integrable over $\mathbb{R}$, while $\mathcal{K}$ was clearly not, the replacement of $S_{p}(\omega)$ by $S_{p}(\bar{\omega})$ might be considered. This would provide a first approximation of the contribution of one peak. This discussion is further pursed in the sequel. Meanwhile, as the derivation of a local approximation such as (31) is not always obvious, as $\mathcal{K}$ might exhibit a more complex form, we systematize the development of a local approximation that could be obtained regardless of the complexity of $\mathcal{K}$, under the computational limits however of a symbolic calculus tool.

More generally thus, another family of approximations is for instance obtained by considering the Laurent series expansion of $f_{1}$ in (27) (which is available in common symbolic calculus packages), for small damping coefficient. This requires the Taylor series expansion of the power spectral density of the loading

$$
\begin{aligned}
S_{p}[\bar{\omega}(1+\xi \eta)] & =\sum_{i=0}^{+\infty} \frac{(\xi \eta \bar{\omega})^{i}}{i !} S_{p}^{(i)}(\bar{\omega}) \\
& =S_{p}(\bar{\omega})+\xi \eta \bar{\omega} S_{p}^{\prime}(\bar{\omega})+\operatorname{ord}\left(\xi^{2}\right)
\end{aligned}
$$

as well as the series expansion of $\mathcal{K}$

$$
\begin{aligned}
\mathcal{K} & =\sum_{i=0}^{+\infty} \frac{\xi^{i}}{i !} \mathcal{K}^{(i)}(0) \\
& =\frac{1}{k^{2} \xi^{2}} \frac{1}{4\left(1+\eta^{2}\right)}-\frac{1}{k^{2} \xi^{2}} \frac{\eta\left(2+\eta^{2}\right)}{4\left(1+\eta^{2}\right)^{2}} \xi+\operatorname{ord}\left(\xi^{2}\right)
\end{aligned}
$$

where the superscript $(i)$ denotes the $i$ th derivative. At leading order, the local approximation is thus the same as that obtained by assuming $\eta=\operatorname{ord}(1)$ and $\xi \ll 1$ as before. It is however specific to this problem that the smallness of $\xi$ is associated with the local behavior of $\mathcal{K}$ for $\eta \ll 1$, as these two ratios appear in a homogenous way in the leading terms. This might not be a general fact, as shown with problem 4 . The series in (32) is usually truncated after the leading order $\xi^{0}$, assuming that the power spectral density of the loading does not vary significantly across the resonance peaks. This assumption might be relaxed easily with the multiple timescale spectral analysis. This option is detailed later.

Keeping only leading order terms in both series expansions yields

$\tilde{f}_{1}[\omega(\eta)] d \omega=\frac{S_{p}(\bar{\omega})}{k^{2}} \frac{\bar{\omega}}{4 \xi\left(1+\eta^{2}\right)} d \eta$

which matches the residual $f_{1}$ around $\eta=0$ and decreases fast enough in the far field to ensure integrability, see curve with white dots in Fig. 3(c). Integration of (34) provides the resonant contribution associated with the peak at $\omega=\bar{\omega}$

$k_{2, r 1}=\int_{-\infty}^{+\infty} \frac{S_{p}(\bar{\omega})}{k^{2}} \frac{\bar{\omega}}{4 \xi\left(1+\eta^{2}\right)} d \eta=\frac{S_{p}(\bar{\omega})}{k^{2}} \frac{\pi \bar{\omega}}{4 \xi}$.

The next residual follows after subtraction of the approximation $\tilde{f}_{1}$ from the first residual, $f_{2}=f_{1}-\tilde{f}_{1}$ where $f_{1}$ and $\tilde{f}_{1}$ are given in (29) and (34) respectively. Although the resulting expression is rather heavy, the same analysis of the local contributions to the integral reveals the existence of a major contribution from the peak located in $\omega=-\bar{\omega}$. Unsurprisingly, after the introduction of a strained coordinate and local approximation, that local contribution amounts to $k_{2, r_{2}}=k_{2, r 1}$ so that finally the total resonant contribution is obtained as

$k_{2, r}=k_{2, r_{1}}+k_{2, r_{2}}=\frac{S_{p}(\bar{\omega})}{k^{2}} \frac{\pi \bar{\omega}}{2 \xi}$.

A formal application of the methodology presented in Section 3 thus provides the background and resonant contributions given in (26) and (36). They are exactly the same as those derived by Davenport [38]. This indicates that his intuitive replacement of the actual power spectral density of loading by a constant value corresponds to the leading order solution of the multiple scales approach. Actually, with our notations, his method consists in considering 
$\tilde{f}_{1}(\omega)=S_{p}(\bar{\omega})|H(\omega)|^{2}$

as a first approximation of $f_{1}$. It is obtained by assuming the replacement of $S_{p}$ by a constant value $S_{p}(\bar{\omega})$ and a shift in the kernel function. It is interesting to notice that this latter operation is mandatory to ensure the far-field integrability. Also interesting is the adequacy of that "local" approximation to capture both peaks at the same time. This local approximation is represented with a thick dashed line in Fig. 3(c).

As hinted by the series approximations (32) and (33), the derivation of the approximation is not unique. For instance, it is readily seen that the first-order correction terms in $\xi$ could offer a more accurate representation of the variation of the power spectral density of the loading across the resonance peaks, e.g. consideration of a linearly varying spectral density rather than a constant value. To be consistent, the first two terms could thus be kept in the series (32) and (33). Unfortunately, in the far field, the power spectral density of the loading would thus behave as $\xi \eta$, while $\mathcal{K}$ behaves as $\xi \eta^{-1}$, so that their product is not integrable at infinity. This is thus not a good option and solutions are discussed later.

Meanwhile, alternatively to (33), we develop another local approximation for the rational fraction in $f_{1}$. It is based on the $(p, q)$ Padé approximants [55] which are the best rational fractions, with given polynomial degrees $p \geq 0$ and $q \geq 0$ in the numerator and the denominator respectively, that approximate an analytical function. In our case, the function to be approximated is already a rational fraction, but the consideration of its Padé approximants, with other degrees than the actual ones, provides approximants with properties in the far field that might be tailored to the needs of the derivation. For integrability reasons, the condition $m \geq n+2$ has to be fulfilled, at least. The simplest Padé approximation is thus the $(0,2)$ one and a first alternative to (33) thus reads

$\mathcal{P}_{0,2}[\mathcal{K}]=\frac{1}{4} \frac{\left(1-4 \xi^{2}\right)^{3}}{\eta^{2}\left(12 \xi^{4}-3 \xi^{2}+1\right)+2 \xi \eta\left(1-4 \xi^{2}\right)+\left(1-4 \xi^{2}\right)^{2}}$.

This local approximation of the kernel might be combined with any approximation of the power spectrum of the loading, for which the Talyor series (32) is however preferred as it only involves a very limited number of function estimates. For integrability reasons again, the series expansion on the loading has to be truncated at the leading order term $S_{p}(\bar{\omega})$ when combined with the $(0,2)$ Padé approximant of $\mathcal{K}$, which finally yields, after multiplication by a factor 2 in order to account for the peak located in $\omega=-\bar{\omega}$

$k_{2, r}=\frac{S_{p}(\bar{\omega})}{k^{2}} \frac{\pi \bar{\omega}}{2 \xi} \frac{\left(1-4 \xi^{2}\right)^{2}}{\sqrt{1-4 \xi^{2}+12 \xi^{4}}}=\frac{S_{p}(\bar{\omega})}{k^{2}} \frac{\pi \bar{\omega}}{2 \xi}\left[1+6 \xi^{2}+\operatorname{ord}\left(\xi^{4}\right)\right]$.

The final result is very similar to (36). Because the approximation in (38) has no restriction on the smallness of the damping coefficient, this alternative solution is perhaps more appropriate in case of moderate viscous damping ( $\xi \simeq 0.1$ ), while (36) was only valid for small damping. Nevertheless, this enrichment of the total solution is rarely useful for a moderate viscous damping as, in such a case, the background contribution is usually leading.

As long as the power spectral density of the loading is approximated by a constant value across the resonance peak, there is no big advantage in using Padé approximants to derive a new approximation, except perhaps that the approximation could be obtained with an automated symbolic calculus software. However, it is an ideal tool to cope with higher order approximations of the loading. Indeed, adapting the degrees in the Pade approximant of the kernel may compensate for a higher order approximation of the power spectral density of the loading across the peak. For instance, the $(0,4)$ Padé approximant of the kernel reads

$\tilde{\mathcal{K}}=\frac{1}{4} \frac{1}{1+2 \eta \xi+\eta^{2}+\eta^{3} \xi+\frac{17}{4} \eta^{4} \xi^{2}}$

(which has been simplified on account that $\xi^{2} \ll 1$ ), see Fig. 3(c), and might be used in conjunction with approximations of the loading up to the second order. Applying the same calculus as before finally yields

$k_{2, r}=\frac{1}{k^{2}}\left(S_{p}(\bar{\omega})+\frac{\xi}{4} \bar{\omega} S_{p}^{\prime}(\bar{\omega})-\frac{\xi}{4} \bar{\omega}^{2} S_{p}{ }^{\prime \prime}(\bar{\omega})\right) \frac{\pi \bar{\omega}}{2 \xi}$

where, again, the damping ratio $\xi$ is assumed to be small. Comparison of the orders of magnitude of the different terms in the brackets should be used in practical applications to determine whether the slope and the curvature of the spectral density of the loading need to be included in the approximation.

Besides the refinement of the resonant contribution, the multiple timescale spectral analysis method may also be used to refine the background component. Indeed, another way to improve the local behavior around $\omega \simeq 0$ is to consider

$\tilde{\mathbf{K}}=\frac{1}{k^{2}}\left(1+2\left(1-2 \xi^{2}\right) \frac{\omega^{2}}{\bar{\omega}^{2}}+\operatorname{ord}\left(\frac{\omega^{4}}{\bar{\omega}^{4}}\right)\right)$

so that the background component is composed of the classical quasi-static component as well as a quasi-kinematic component

$k_{2, b}=\frac{k_{2, p}}{k^{2}}+\frac{2 k_{2, \dot{p}}}{k^{2} \bar{\omega}^{2}}$

where $k_{2, \dot{p}}=\int_{-\infty}^{+\infty} \omega^{2} S_{p}(\omega) d \omega$ represents the cumulant of the time derivative of the loading, provided it exists. This advanced modeling of the background component should be considered when the timescale separation between the loading and the system is moderate $(\varepsilon \lesssim 0.1)$, rather than small $(\varepsilon \ll 1)$. The resonant contributions remain, after subtracting both the quasi-static and the quasi-kinematic contributions, the same as those obtained earlier. The new background approximation (43) might thus be combined with any resonant contribution (36) or (41).

\subsection{Problem 2: variance of the response velocity}

As a second problem, we formalize the derivation of an approximate solution for the response velocity of the oscillator considered in problem 1. Its cumulant is given by

$k_{2}=\int_{-\infty}^{\infty} S_{p}(\omega) \omega^{2}|H(\omega)|^{2} d \omega$.

In dedicated literature and codes, the solution of this problem is usually formulated as a resonant component only. It is justified by the highpass nature of the velocity response transfer $\mathbf{K}=\omega^{2}|H(\omega)|^{2}$. In order to demonstrate again the applicability of the general method presented in Section 3, this problem is treated with the proposed framework. Its extension to higher derivatives is straightforward although not presented in this document.

The background component of the response is obtained by approximating the kernel as $\tilde{\mathbf{K}}=\omega^{2} / k^{2}$, so that the local approximation of the integrand in the low-frequency range is $\tilde{f}=\left(\omega^{2} / k^{2}\right) S_{p}(\omega)$, see Fig. $4(\mathrm{a})$, and the background component of the response reads

$k_{2, b}=\frac{1}{k^{2}} \int_{-\infty}^{+\infty} \omega^{2} S_{p}(\omega) d \omega=\frac{k_{2, \dot{p}}}{k^{2}}$

where $k_{2, \dot{p}}$ represents the cumulant of the time derivative of the loading, provided it exists. If not, this background component 
should be discarded. Loading processes that are not differentiable in a mean square sense are encountered in wind engineering application, e.g. the point-like wind velocity turbulence, but are usually low-pass filtered as a result of spatial coherence [56,57]. Subtracting off the approximation $\tilde{f}$ from the initial power spectrum of the response velocity provides the first residual

$f_{1}(\omega)=S_{p}(\omega) \frac{\omega^{2}}{k^{2} \xi^{2}} \mathcal{K}(\omega)$

with the same $\mathcal{K}$ as that defined in (30). Similar to the approach adopted for problem 1, an analysis of the contributions to the integral highlights the existence of two peaks with equal contributions. The introduction of the stretched coordinate $\eta$ as defined in (28) yields

$f_{1}[\omega(\eta)] d \omega=S_{p}[\bar{\omega}(1+\xi \eta)] \frac{\bar{\omega}^{2}(1+\xi \eta)^{2}}{k^{2} \xi^{2}} \mathcal{K}(\eta, \xi) \xi \bar{\omega} d \eta$

from which it is readily seen that the only difference with (29) concerns the numerator $\bar{\omega}^{2}(1+\xi \eta)^{2}$, approximated as $\bar{\omega}^{2}$ at the leading order. Following the same analysis as before, the total resonant contribution finally reads

$k_{2, r}=\frac{S_{p}(\bar{\omega})}{k^{2}} \frac{\pi \bar{\omega}^{3}}{2 \xi}$.

This resonant contribution corresponds to the usual approximation. The multiple timescale spectral analysis thus recovers the classical solution, with the major difference that it also brings to light a quasi-kinematic component (45), that is usually small but could dominate in some cases of highly damped structures.

\subsection{Problem 3: covariance of modal amplitudes}

The next problem illustrates the applicability of the general concept to a linear MDOF structure. In wind engineering, Davenport's approximation has opened the door to a very fast and accurate estimation of the variance of the response of a single oscillator, i.e. modal responses in a MDOF system. The approximation could thus be used in the framework of an SRSS (square root of the sum of the squares) recombination of the modal responses, as there were no means to estimate the covariances of modal responses in a similarly accurate and efficient manner. Such estimations were developed independently by $\mathrm{Gu}$ [58] and Denoël [42] in an intuitive manner, by replacing the cross-power spectral density of the modal loading by a white noise. Although the resulting approximation was intensively checked and validated by comparison with heavier numerical integration, there is no sound

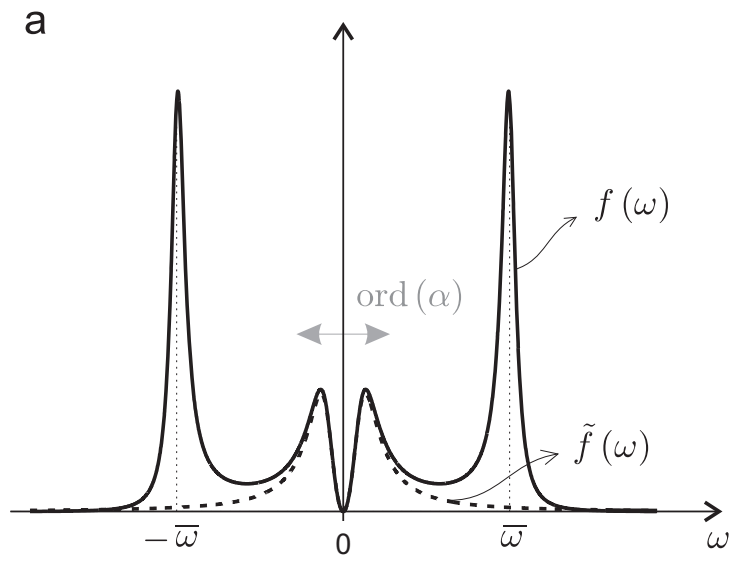

assessment of the limitations of the method. This is now done with the general framework of the multiple timescale spectral analysis.

The covariance of modal amplitudes in modes $m$ and $n$ is given by

$k_{2}=\int_{-\infty}^{\infty} S_{p}(\omega) H_{m}(\omega) H_{n}^{*}(\omega) d \omega$

where $S_{p}(\omega)$ represents the cross-power spectral density of the modal loading and

$H_{m}(\omega)=\frac{1}{k_{m}} \frac{1}{1-\left(\frac{\omega}{\bar{\omega}_{m}}\right)^{2}+2 \mathrm{i} \xi \frac{\omega}{\bar{\omega}_{m}}}$

where $k_{m}, \bar{\omega}_{m}$ and $\xi$ are the generalized stiffness, the circular natural frequency and the damping ratio in mode $m$, respectively. The frequency response function $H_{n}(\omega)$ in mode $n$ is obtained by replacing $m$ by $n$ in the above definitions. The generalized stiffness and the natural frequency are denoted by $k_{n}$ and $\bar{\omega}_{n}$, respectively, while the same damping coefficient $\xi$ is considered, for the conciseness of the illustration. The problem at hand is thus also a particular case of (2), where the kernel function $\mathbf{K}\left(\boldsymbol{\omega}_{j}\right)$, with $\omega_{j} \equiv \omega$, reads

$\mathbf{K}\left(\omega_{j}\right)=H_{m}(\omega) H_{n}^{*}(\omega)$.

In typical applications, the fast decrease of the cross-power spectral density $S_{p}(\omega)$ is such that the product $S_{p}(\omega) K(\omega)$ exhibits on $\omega \in[0 ;+\infty]$ either one peak, if the response is essentially background or resonant, or two peaks (one background and one resonant, or two resonant ones), or three peaks (one background and two resonant), see Fig. 5(a, c, and e). Application of the general concept of Section 3 allows the derivation of an approximation that covers these three cases at once, while providing a very accurate estimation of the modal covariance. This consequently offers the possibility to combine inexpensively the modal responses in a complete quadratic $\mathrm{CQC}$ manner.

The background component of the response results from the low frequency loading. It is obtained by approximating the kernel locally by $\tilde{\mathbf{K}}=1 / k_{m} k_{n}$, so that

$k_{2, b}=\frac{1}{k_{m} k_{n}} \int_{-\infty}^{\infty} S_{p}(\omega) d \omega=\frac{\sigma_{p m n}}{k_{m} k_{n}}$

where $\sigma_{p_{m n}}$ represents the covariance of the modal loading. Subtracting off the approximation $\tilde{f}(\omega)=\left(1 / k_{m} k_{n}\right) S_{p}(\omega)$ from the initial function, we obtain a first residual

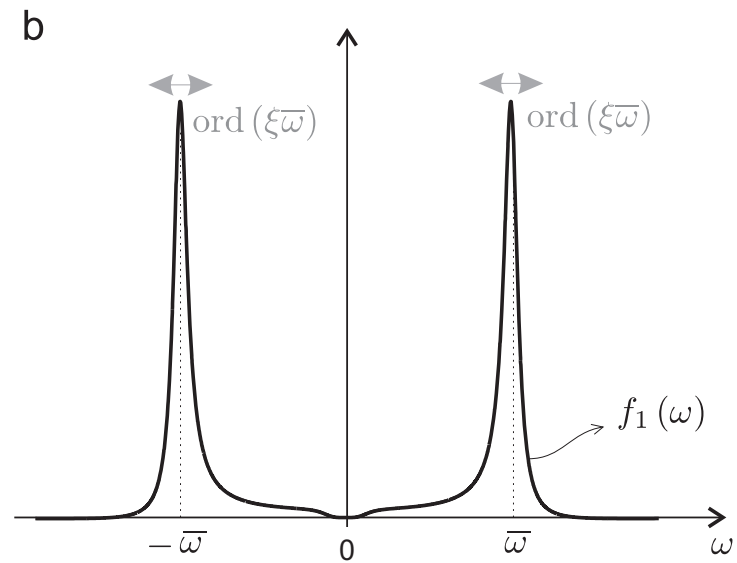

Fig. 4. Problem 2. (a) Power spectral density of the velocity response and its background approximation $\tilde{f}$, and (b) remainder $f_{1}(\omega)$. 

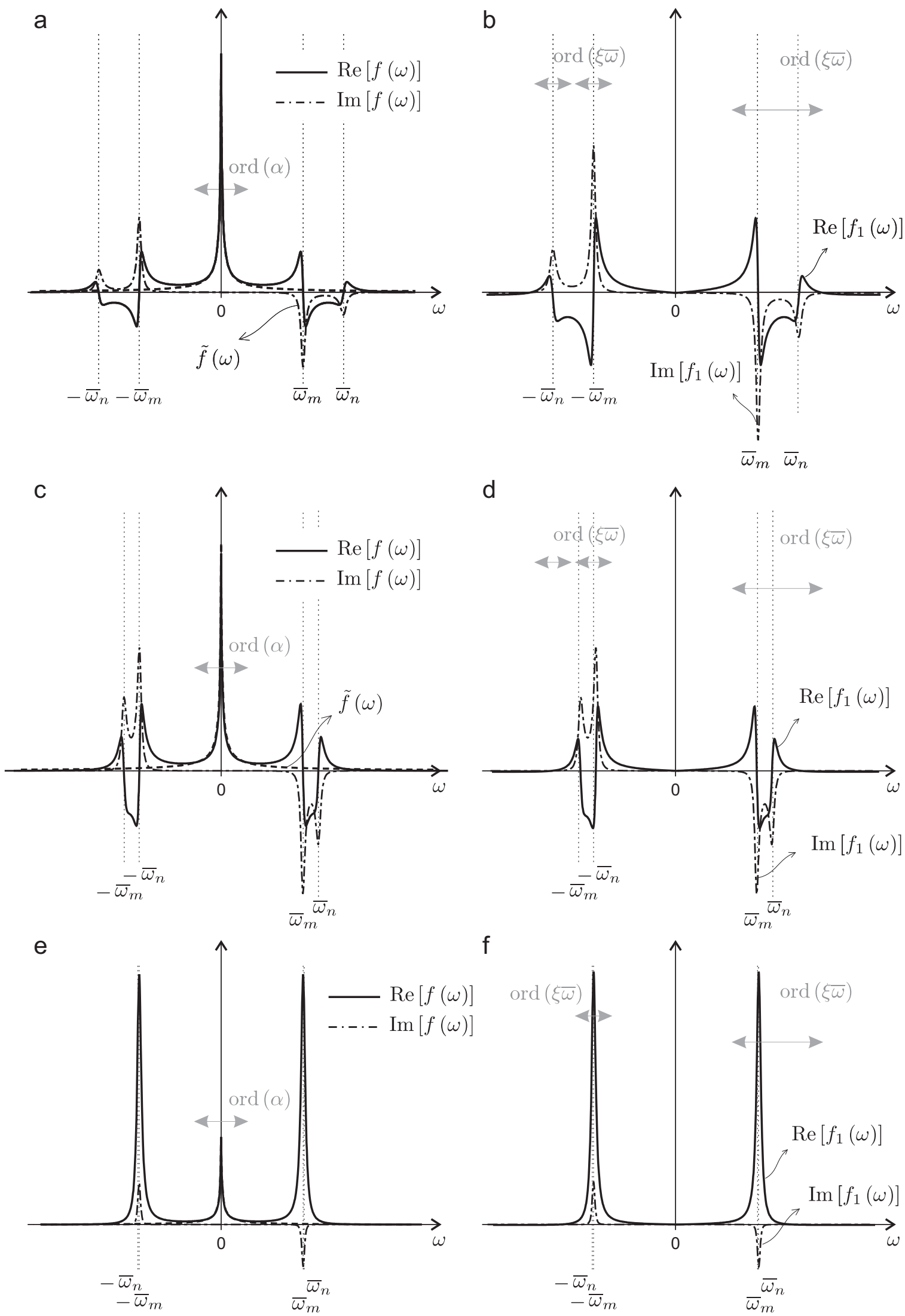

f

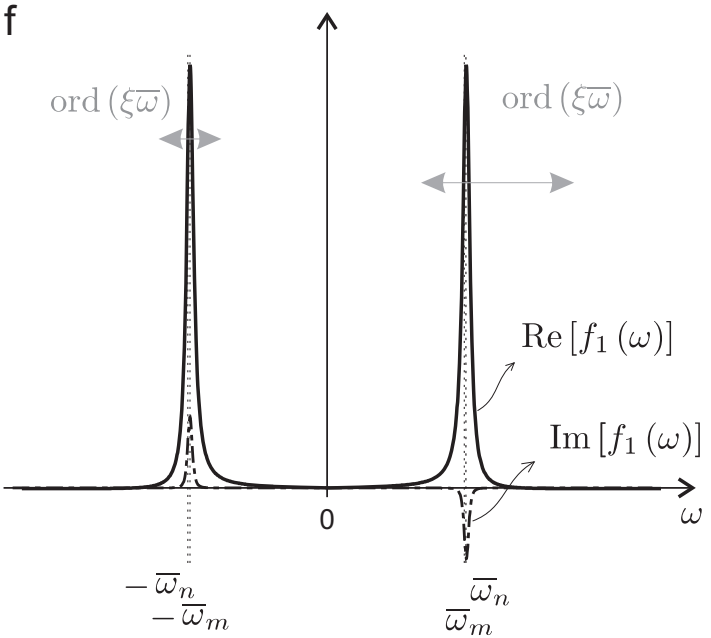

Fig. 5. Problem 3. (a, c, and e) Power spectral density of the response and its background approximation $\tilde{f}$, and (b, d, and f) remainder $f_{1}(\omega)$. The three examples correspond to different values of the relative distance between the two natural frequencies: $\left(\bar{\omega}_{n} / \bar{\omega}_{m}=1.5, \varepsilon=0.2\right),\left(\bar{\omega}_{n} / \bar{\omega}_{m}=1.2, \varepsilon=0.0909\right)$ and $\left(\bar{\omega}_{n} / \bar{\omega}_{m}=1.005, \varepsilon=0.00249\right)$. The damping ratio used for the illustration is $\xi=0.03$ in both modes. 
$f_{1}(\omega)=S_{p}(\omega)(\tilde{\mathbf{K}}(\omega)-\tilde{\mathbf{K}})=S_{p}(\omega)\left(H_{m}(\omega) H_{n}^{*}(\omega)-\frac{1}{k_{m} k_{n}}\right)$.

This residual is characterized by the absence of background component in the low frequency range. It is represented in Fig. 5(b, d, and f) for $\xi=0.03$ and $\bar{\omega}_{n} / \bar{\omega}_{m}=1.5,1.2$ and 1.005. The integral of $f_{1}$ provides, with no assumption, the exact supplement to the background component, in order to recover the exact covariance. It might contain one or two peaks on $\omega \in[0,+\infty]$, depending on the relative distance between the two natural frequencies, see Fig. 5. This distance is measured by the dimensionless number $\varepsilon \in[-1 ; 1]$

$\varepsilon=\frac{\bar{\omega}_{n}-\bar{\omega}_{m}}{\bar{\omega}_{n}+\bar{\omega}_{m}}$

and the peaks are distinct if $\varepsilon \gtrsim \xi$. This number is respectively equal to $0.200,0.0909$ and 0.00249 for the three cases represented in Fig. 5(b, d, and f).

There are several ways to develop local approximations for (53). One approach consists in focusing on the two peaks at the same time, an option that was already considered in [42]. This is formally written by defining the new coordinate $\eta$ as

$\omega=\frac{\bar{\omega}_{m}+\bar{\omega}_{n}}{2}+\eta\left(\bar{\omega}_{n}-\bar{\omega}_{m}\right)=\frac{\bar{\omega}_{m}+\bar{\omega}_{n}}{2}(1+2 \varepsilon \eta)$,

which is thus such that it covers a frequency range centered on $\left(\bar{\omega}_{m}+\bar{\omega}_{n}\right) / 2$ and also such that both peaks are located inside the unit disk $|\eta| \lesssim 1$. To simplify the notations, we introduce

$\mathcal{K}(\eta)=H_{m}[\omega(\eta)] H_{n}^{*}[\omega(\eta)]-\frac{1}{k_{m} k_{n}}$

in which (55) is readily substituted. This provides a rather rich expression for $\mathcal{K}(\eta)$

$\mathcal{K}(\eta)=\frac{1}{k_{m} k_{n}} \frac{(1+2 \eta \varepsilon) \mathcal{N}_{1}}{\mathcal{D}_{1}^{2}-\mathcal{D}_{2}^{2}}$

where

$$
\begin{aligned}
\mathcal{N}_{1}= & -4 \xi^{2}-2 \varepsilon\left(\eta+4 \mathrm{i} \xi+4 \eta \xi^{2}\right)+2 \varepsilon^{2}\left(1-6 \eta^{2}-8 \mathrm{i} \eta \xi+2 \xi^{2}\right) \\
& +4 \varepsilon^{3}\left(\left(1-2 \eta^{2}\right) \eta+\left(1-4 \eta^{2}\right) \mathrm{i} \xi+2 \eta \xi^{2}\right), \\
\mathcal{D}_{1}= & 4 \varepsilon^{2} \eta^{2}+4 \eta \varepsilon+4 \mathrm{i} \eta \xi \varepsilon^{2}-\varepsilon^{2}+2 \mathrm{i} \xi \varepsilon \\
\mathcal{D}_{2}= & 2 \varepsilon-2 \mathrm{i} \xi-4 \mathrm{i} \eta \xi \varepsilon
\end{aligned}
$$

and whose real part is represented by a solid line in Fig. 6. Thanks to the change of variable, the two peaks that were located in the positive frequency range are now located in the unit disk $|\eta| \lesssim 1-$ they are clustered or not, depending on the condition $\varepsilon \gtrsim \xi-$, while the peaks that were located in the negative frequency range are repelled toward minus infinity (if $\bar{\omega}_{n}>\bar{\omega}_{m}$; toward plus infinity otherwise). Their central position in the stretched coordinate system is readily obtained as the solution of

$-\frac{\bar{\omega}_{m}+\bar{\omega}_{n}}{2}=\frac{\bar{\omega}_{m}+\bar{\omega}_{n}}{2}+\eta\left(\bar{\omega}_{n}-\bar{\omega}_{m}\right)$,

i.e. $\eta=-\varepsilon^{-1}$. The actual repulsion of these peaks towards infinity thus requires discussion on the smallness of $\varepsilon$. Fig. 6(a) indicates

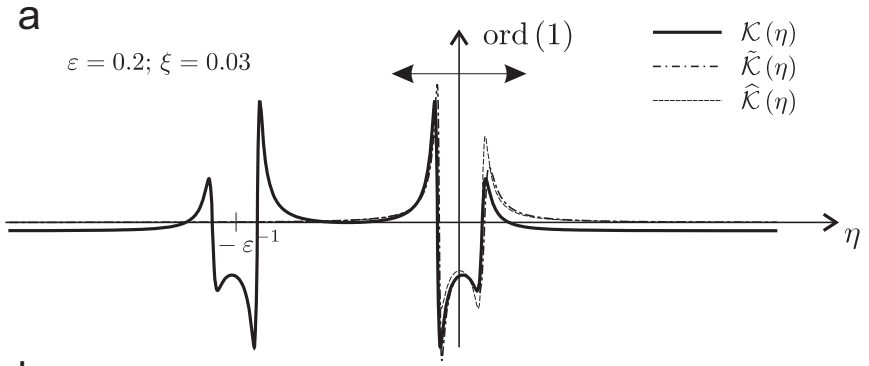

b
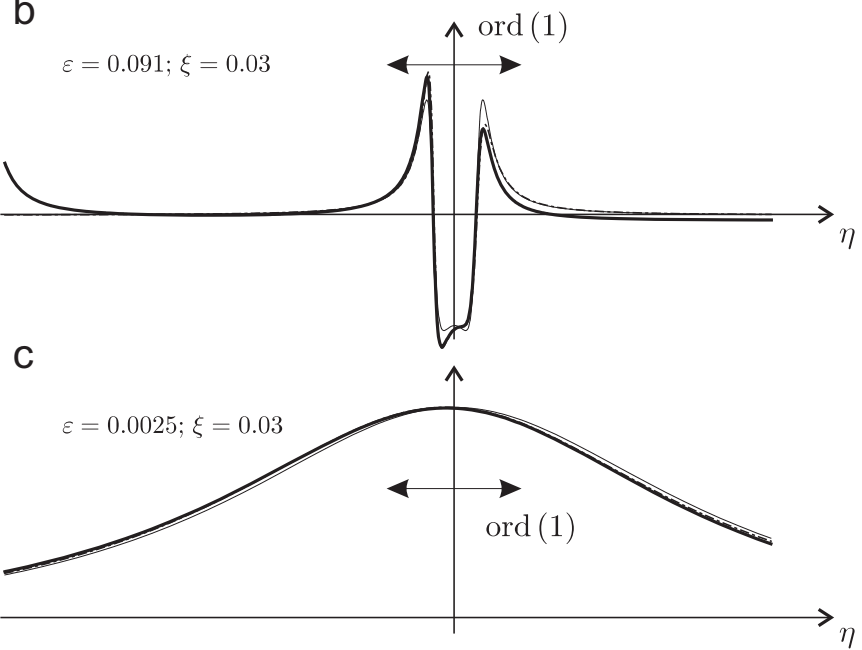

Fig. 6. Problem 3. Approximations of the factor related to the structural behavior $\mathcal{K}$ in the remainder $f_{1}$. Plots are given in the stretched coordinate system centered on the mean natural frequency and spanning both peaks of the positive frequency domain. When $\varepsilon \ll \xi$, see (c), the considered stretching does not localize the contribution into a unit disk $|\eta| \lesssim 1$. When $\varepsilon$ is not small compared to 1 , the peaks that were in the negative frequency range and not repelled to infinity, see (a).

that a relative distance as large as $\varepsilon=0.2$ still splits up the two pairs of peaks. Larger values of $\varepsilon$, which correspond to cases where there is at least one order of magnitude difference in the natural frequencies $\bar{\omega}_{m}$ and $\bar{\omega}_{n}$ shall be considered with care as the peaks that were initially in the negative frequency range are still in the unit disk $|\eta| \lesssim 1$ and the development of a local approximation might prove inaccurate. This observation shows that this first stretching of the problem coordinates is mainly appropriate when the peaks are close (but not necessarily merged), i.e. $\varepsilon^{2} \ll 1$. This is well the underlying idea in the stretching (55) which aims at catching two of the four possible peaks at the same time, independently of their width, as the stretching does not involve $\xi$.

The real part of $\mathcal{K}$ features non-zero asymptotes as indicated by (57), $\lim _{\eta \rightarrow \pm \infty} \mathcal{K}=-1$. Neglecting the high order terms in $\eta$ especially those of the denominator which are responsible for the existence of the undesired peaks in the far field, and at the same time assuming $\xi^{2} \ll 1$, we obtain a first local approximation of $\mathcal{K}$. The degree of the denominator is deliberately dropped from four to two in order to capture the possible existence of two poles in the unit disk, while discarding the other two poles that were repelled toward minus infinity. The degree of the numerator is then decreased down to zero in order to ensure integrability and boundedness in the far field. This local approximation reads

$\tilde{\mathcal{K}}[\omega(\eta)]=\frac{1}{k_{m} k_{n}} \frac{1+2 \varepsilon^{2}+4 \mathrm{i} \xi\left(-2 \varepsilon+\varepsilon^{3}\right)}{8 \varepsilon^{2}\left(-\varepsilon^{2}+6 \mathrm{i} \xi \varepsilon+2\right) \eta^{2}+8 \varepsilon\left(-\mathrm{i} \xi \varepsilon^{3}-\varepsilon^{2}+4 \mathrm{i} \xi \varepsilon+2 \xi^{2}\right) \eta+\left(\varepsilon^{4}-4 \mathrm{i} \xi \varepsilon^{3}-4 \varepsilon^{2}+8 \mathrm{i} \xi \varepsilon+4 \xi^{2}\right)}$ 
which is actually very similar to the $(0,2)$ Padé approximant of $\mathcal{K}(\omega)$ - that a symbolic calculus software would provide -, the main difference pertaining to the terms that are dropped on account that $\xi^{2} \ll 1$. Cases of interest are those where $\varepsilon^{2} \ll 1$, not meaning that the natural frequencies are close, or that the peaks are coalescent, but simply that the natural frequencies are not several orders of magnitude away from each other. Two cases are of particular relevance: in the case $0 \lesssim \varepsilon \lesssim \xi$, the peaks are coalescent (Fig. 6(c)), in the case $\xi \lesssim \varepsilon \ll 1$, they are well separated (Fig. 6(a and b)).

Under the sole restriction $\varepsilon^{2} \ll 1$, which thus covers these two cases, the approximation further simplifies to

$\widehat{\mathcal{K}}[\omega(\eta)]=\frac{1}{4 k_{m} k_{n}} \frac{1}{\left(4 \eta^{2}-1\right) \varepsilon^{2}+2 \mathrm{i} \xi \varepsilon+\xi^{2}}$.

It is represented by thin lines in Fig. 6. This approximation presents the required properties of locality, local accuracy and global integrability, while keeping a very simple explicit formulation. The simplicity of this expression makes it clear that there exist two poles when $\varepsilon \gg \xi$, while the kernel behaves as a constant when $\varepsilon \ll \xi$.

The derivation of an approximate solution also requires a development for $S_{p}(\omega)$ in (53). With the same objective to keep the number of function estimates as low as possible, a Taylor series expansion around $\eta=0$ appears as an adequate solution. In the stretched coordinate system, we thus write

$S_{p}(\omega(\eta))=S_{p}\left(\frac{\bar{\omega}_{m}+\bar{\omega}_{n}}{2}\right)+\left(\bar{\omega}_{n}-\bar{\omega}_{m}\right) \eta S_{p}^{\prime}\left(\frac{\bar{\omega}_{m}+\bar{\omega}_{n}}{2}\right)+\cdots$.

Truncating this series after the first term, the local contribution resulting from the resonant peaks in the positive frequency domain is

$k_{2, r 1}=\frac{1}{k_{m} k_{n}} S_{p}\left(\frac{\bar{\omega}_{m}+\bar{\omega}_{n}}{2}\right) \frac{\omega_{n}+\omega_{m}}{2} \frac{\pi(\xi-\mathrm{i} \varepsilon)}{4\left(\varepsilon^{2}+\xi^{2}\right)}$.

In this expression, we suggest to replace $S_{p}\left(\left(\bar{\omega}_{m}+\bar{\omega}_{n}\right) / 2\right)$ by $\frac{1}{2}\left(S_{p}\left(\bar{\omega}_{m}\right)+S_{p}\left(\bar{\omega}_{n}\right)\right)$ which is much more efficient since the cross power spectral density of the loading is thus only estimated for the whole set of natural frequencies, rather than the average of all possible combinations. This option was already discussed in [42]. With the second pair of peaks located in the negative frequency range, which provides the complex conjugate of contribution $k_{2, r 1}$, the total resonant contribution thus reads

$k_{2, r}=\frac{1}{k_{m} k_{n}} \operatorname{Re}\left[\frac{S_{p}\left(\bar{\omega}_{m}\right)+S_{p}\left(\bar{\omega}_{n}\right)}{2} \frac{\omega_{n}+\omega_{m}}{2} \frac{\pi(\xi-\mathrm{i} \varepsilon)}{2\left(\varepsilon^{2}+\xi^{2}\right)}\right]$.

This approximation remarkably converges towards (36) as $\bar{\omega}_{m} \rightarrow \bar{\omega}_{n}$, i.e $\varepsilon \rightarrow 0$. This expression also shows that the imaginary-to-real ratio of the integrated kernel is given by $\varepsilon / \xi$. As soon as the natural frequencies are further away than the damping ratio, relatively, the response is thus mainly governed by the imaginary part of the loading, should it exist.

This result agrees very well with the results given in [42] which were however limited to real cross power spectral loading, but also with those given in [58]. This indicates that the only assumptions in these two other developments are well related to the distance between the natural frequencies.

\subsection{Problem 4: third moment of the response}

The objective of this fourth problem is doublefold: to illustrate applicability to higher order cumulants of the response, and to illustrate the degeneracy of the integral multiplicity. The third cumulant of the response of a simple oscillator subjected to a stationary excitation is given by $k_{3}=\iint_{-\infty}^{\infty} B_{p}\left(\omega_{1}, \omega_{2}\right) H\left(\omega_{1}\right) H\left(\omega_{2}\right) H^{*}\left(\omega_{1}+\omega_{2}\right) d \omega_{1} d \omega_{2}$

where $B_{p}\left(\omega_{1}, \omega_{2}\right)$ represents the bispectrum of the loading and $H$ stands for the frequency response function, as introduced in (50). An investigative version of the multiple timescales spectral analysis was presented in [43], where more details and examples of applications may be found. The following exposé is therefore deliberately kept short and limited to pinpointing important steps of the approximation.

Fig. 7(a and c) sketches a typical bispectrum of loading, as could be encountered in wind engineering applications, as a result of the quadratic transformation of the wind turbulence in the process of the establishment of quasi-steady wind pressures. In this case, the characteristic frequency of the loading $\alpha$ is not only isotropic as explained earlier $\left(\alpha_{2}=\alpha_{3}:=\alpha\right)$, but more importantly much smaller than the natural frequencies of the system. These correspond to the locations of the peaks in the kernel function $\mathbf{K}\left(\boldsymbol{\omega}_{j}\right):=H\left(\omega_{1}\right) H\left(\omega_{2}\right) H^{*}\left(\omega_{1}+\omega_{2}\right)$ as represented in Fig. 7(b and d). This function exhibits, in particular, six biresonance peaks which are responsible for the possible dynamic (de-)amplification of the third-order statistics. The product of $B_{p}\left(\omega_{1}, \omega_{2}\right)$ and $\mathbf{K}\left(\omega_{1}, \omega_{2}\right)$ was actually given in Fig. 1. Significant contributions to the integral are at most, one peak in the neighborhood of the origin, plus six additional peaks corresponding to the biresonance phenomenon. An intuitive replacement of the bispectrum of the loading by a constant value, as was done at the second order, does not provide a suitable approximation, as extensively discussed in [43]. This justifies the need for a method as the multiple timescale one.

The background response, in the low frequency range, is simply obtained with the local approximation $\tilde{\mathbf{K}}\left(\omega_{1}, \omega_{2}\right)=1 / k^{3}$, such that

$k_{3, b}=\frac{1}{k^{3}} \iint_{-\infty}^{+\infty} B_{p}\left(\omega_{1}, \omega_{2}\right) d \omega=\frac{k_{3, p}}{k^{3} .}$

where $k_{3, p}$ represents the third cumulant of the loading. Subtracting off the first approximation $\tilde{\mathbf{K}}\left(\omega_{1}, \omega_{2}\right) B_{p}\left(\omega_{1}, \omega_{2}\right)$ from the initial function returns the residual

$f_{1}\left(\omega_{1}, \omega_{2}\right)=B_{p}\left(\omega_{1}, \omega_{2}\right)\left(\mathbf{K}\left(\omega_{1}, \omega_{2}\right)-\tilde{\mathbf{K}}\right)$

whose main contributions result from the six biresonance peaks. For symmetry reasons, only the peak located in $\left(\omega_{1}, \omega_{2}\right)=(\bar{\omega}, 0)$ is considered and the subsequent results are multiplied by a factor 6 . The width of the peaks in both directions is readily shown to be $\xi \bar{\omega}$, so that the closeup view on the peak of interest is obtained with the new coordinates

$\omega_{1}=\bar{\omega}\left(1+\xi \eta_{1}\right) ; \quad \omega_{2}=\xi \bar{\omega} \eta_{2}$

so that the bulk of the peak is spanned as $\eta_{1}$ and $\eta_{2}$ move across the unit disk. The residual

$$
\begin{aligned}
& f_{1}\left[\omega_{1}\left(\eta_{1}\right), \omega_{2}\left(\eta_{2}\right)\right] d \omega_{1} d \omega_{2} \\
& \quad=B_{p}\left[\bar{\omega}\left(1+\xi \eta_{1}\right), \xi \bar{\omega} \eta_{2}\right] \mathcal{K}\left(\eta_{1}, \eta_{2}\right) \xi^{2} \bar{\omega}^{2} d \eta_{1} d \eta_{2}
\end{aligned}
$$

still features all six high peaks, plus some lower additional ones. A Laurent series expansion of the factor $\mathcal{K}$ provides a neat local and bounded approximation of the real part of the kernel

$\tilde{\mathcal{K}}\left(\eta_{1}, \eta_{2}\right)=\frac{1}{k^{3}} \frac{\eta_{1}^{2}+\eta_{1} \eta_{2}+1}{\left(\eta_{1}^{2}+\eta_{1} \eta_{2}+1\right)^{2}+\eta_{2}^{2}}$,

which is much simpler than the original expression of $\mathcal{K}$. Its contour representation is given in Fig. 7(f) and is very similar to the original biresonance peak pattern, given in Fig. 7(d). The other factor in $f_{1}$, related to the bispectrum of the loading, requires a careful attention in the local coordinate frame. Indeed, Fig. 7(e) discloses somewhat parallel contour sets of $B_{p}\left[\omega_{1}\left(\eta_{1}\right), \omega_{2}\left(\eta_{2}\right)\right]$, with strong gradients along 

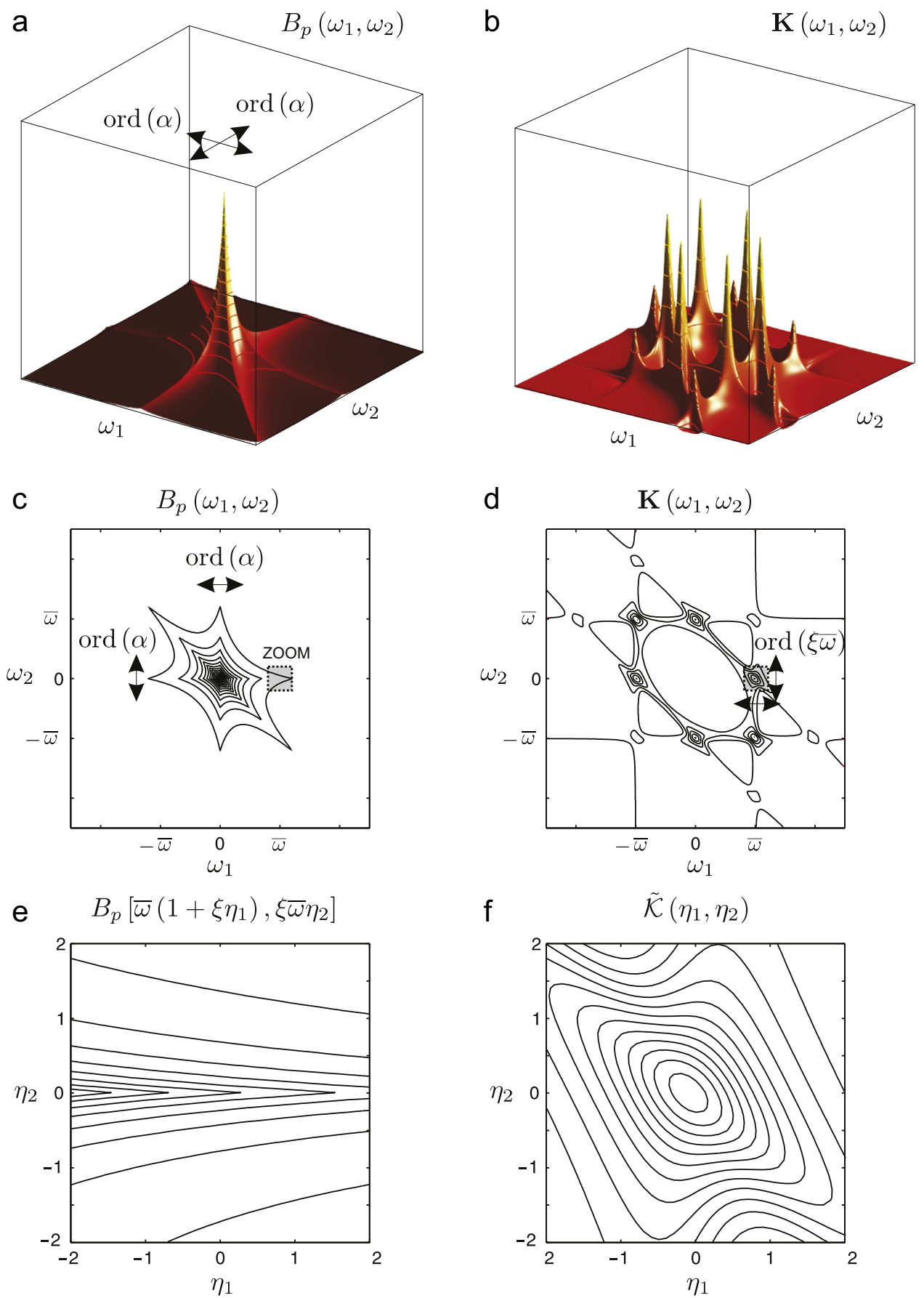

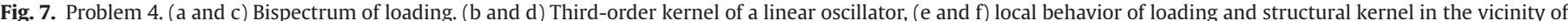

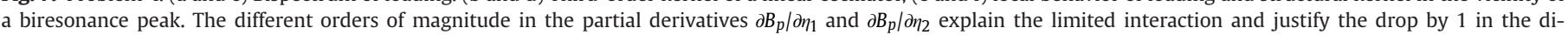
mensionality of integrals.

$\eta_{2}$. This indicates that the dynamics in the loading and in the structure do not interact, locally, along the $\eta_{1}$ coordinate, but well along the $\eta_{2}$ coordinate. It actually translates the interaction of the slow dynamics of the loading and the slow timescale associated with the memory of the system. This justifies why the replacement of the bispectrum by a constant value provides a wrong approximation. Furthermore, it shows that, in the Taylor series approximation,

$$
\begin{aligned}
& B_{p}\left[\bar{\omega}\left(1+\xi \eta_{1}\right), \xi \bar{\omega} \eta_{2}\right] \\
& \quad=B_{p}\left(\bar{\omega}, \xi \bar{\omega} \eta_{2}\right)+\xi \eta_{1} \bar{\omega} \frac{\partial B_{p}\left(\bar{\omega}, \xi \bar{\omega} \eta_{2}\right)}{\partial \omega_{1}}+\operatorname{ord}\left(\xi^{2}\right)
\end{aligned}
$$

similar to (32), only the leading term needs to be retained, for similar reasons as for the second-order analysis. To retain this term provides the required $\eta_{1}$-independence. Finally, integration of the resonant contribution reads

$$
\begin{aligned}
& \iint_{-\infty}^{+\infty} f_{1}\left[\omega_{1}\left(\eta_{1}\right), \omega_{2}\left(\eta_{2}\right)\right] d \omega_{1} d \omega_{2} \\
& \quad=\int_{-\infty}^{+\infty} B_{p}\left(\bar{\omega}, \xi \bar{\omega} \eta_{2}\right) \int_{-\infty}^{+\infty} \tilde{\mathcal{K}}\left(\eta_{1}, \eta_{2}\right) \xi^{2} \bar{\omega}^{2} d \eta_{1} d \eta_{2}
\end{aligned}
$$

where the second integral along $\eta_{1}$ might be worked out in a closed form, so that 
$k_{3, r}=6 \pi \frac{\xi \bar{\omega}^{3}}{k^{3}} \int_{-\infty}^{+\infty} \frac{B_{p}\left(\bar{\omega}, \omega_{2}\right)}{4 \xi^{2} \bar{\omega}^{2}+\omega_{2}^{2}} d \omega_{2}$,

and the total approximation gathering the background and resonant contributions is obtained by adding $k_{3, b}$ from (64) to this expression of $k_{3, r}$. In [43], some illustrations show the good accuracy of this approximation, when the parameters $\alpha$ and $\xi$ involved in the derivation are small.

\subsection{Problem 5: response of a nonlinear system}

The response of a surge platform is governed by an equation of this form

$\ddot{x}+2 \bar{\omega} \xi_{s} \dot{x}+\bar{\omega}^{2} x=\kappa_{d}(U+u-\dot{x})|U+u-\dot{x}|+\kappa_{m} \dot{u}$

where $\bar{\omega}$ and $\xi_{s}$ represent the natural circular frequency and the damping ratio of an SDOF oscillator, respectively, while $\kappa_{\mathrm{d}}$ and the dimensionless number $\kappa_{m}$ are quantities related to the mass and aerodynamic properties of the structure, respectively. According to the linear wave theory, the current is modeled as an average current velocity $U$ and a water-particle velocity $u(t)$, which is modeled as a Gaussian random process with a given power spectral density $S_{u}(\omega)$. One specificity of this problem is that the typical shape of this spectral density is such that the center of gravity of the distribution $\alpha$ is usually much larger than the natural frequency $\bar{\omega}$ of the linear unloaded oscillator [24]. Furthermore, commonly adopted power spectral densities such as the one resulting from the wave height in the Jonswap model [59] are negligible in the low-frequency range, precisely where the fundamental natural frequency of the linear unloaded oscillator is located.

This nonlinear problem might be accurately represented by a second-order Volterra model [34] so that the power spectral density of the response reads

$S_{x}(\omega)=S_{x_{1}}(\omega)+S_{x_{2}}(\omega)$

with

$$
\begin{aligned}
S_{x_{1}}(\omega) & =\left|H_{1}(\omega)\right|^{2} S_{u}(\omega), S_{x_{2}}(\omega) \\
& =2 \int_{\mathbb{R}}\left|H_{2}\left(\omega_{1}, \omega-\omega_{1}\right)\right|^{2} S_{u}\left(\omega_{1}\right) S_{u}\left(\omega-\omega_{1}\right) d \omega_{1} .
\end{aligned}
$$

The first term corresponds to the linearized version of the model, with the first kernel given by

$H_{1}(\omega)=\frac{2 \bar{\omega} \xi_{a}+\kappa_{m} \mathrm{i} \omega}{D(\omega)}$

where $D(\omega)=\bar{\omega}^{2}-\omega^{2}+2 \mathrm{i} \omega \bar{\omega}\left(\xi_{s}+\xi_{a}\right)$ and where the damping coefficient $\xi_{\mathrm{a}}$ results from a fitting of the right-hand side of (72), see [34]. The second term in (73) reflects the nonlinearity in the model, through the second-order kernel defined as

$H_{2}\left(\omega_{1}, \omega_{2}\right)=\frac{a_{2}\left(1-\mathrm{i} \omega_{1} H_{1}\left(\omega_{1}\right)\right)\left(1-\mathrm{i} \omega_{2} H_{1}\left(\omega_{2}\right)\right)}{D\left(\omega_{1}+\omega_{2}\right)}$

with $a_{2}\left(\kappa_{d}, \kappa_{m}\right)$ another coefficient resulting from the replacement of the right-hand side of (72) by a differentiable operator, see [34]. The variance of the response is thus a sum of two terms

$k_{2}=\int_{\mathbb{R}} S_{x_{1}}(\omega) d \omega+\int_{\mathbb{R}} S_{x_{2}}(\omega) d \omega$

which are well two problems of the form (2). In the second term, where $S_{x_{2}}(\omega)$ is given by (74), the variance of the response is thus obtained as a double integral over the frequency space, as a consequence of the nonlinearity of the problem.

The linear part of the response is treated first and separately, as it resembles the examples treated before, with the slight difference that the background component is now inertial rather than quasi-static. In the high frequency range, $\omega \gg \bar{\omega}$, the first kernel might be approximated by $\tilde{H}_{1}(\omega)=-\mathrm{i} \kappa_{m} / \omega$ which is second-order accurate as the remaining terms are multiplied by some coefficients of order $(\bar{\omega} / \alpha)\left(\xi_{s}+\xi_{a}\right)$. A first approximation of $S_{x_{1}}(\omega)$, in the high-frequency range reads

$\tilde{f}_{1}=\left(\frac{\kappa_{m}}{\omega}\right)^{2} S_{u}(\omega)$.

The corresponding background response is

$k_{2, b}=\kappa_{m}^{2} k_{2, \int u}$

with $k_{2, \int u}$ being the variance of the water-particle displacement $\int u d t$, a quantity that is readily obtained by explicit integration of any given water-particle velocity spectrum $S_{u}(\omega)$. Notice the existence of $k_{2, f u}$ requires $S_{u} / \omega^{2}$ to be integrable at $\omega=0$, which is satisfied with common water-particle velocity power spectral densities. Subtracting off the first approximation (78) from $S_{x_{1}}(\omega)$ provides a first residual that might be neglected since $S_{u}(\omega) \simeq 0$ in the neighborhood of $\bar{\omega}$, as justified by the typical shape of wave spectra. This indicates that the low-frequency resonant contribution of the response might not be captured by the linearized version of the system, as well established in the field [60].

The nonlinear part of the response requires a double integration of the product of a kernel $\mathcal{K}:=2\left|H_{2}\left(\omega_{1}, \omega-\omega_{1}\right)\right|^{2}$ and a loading spectrum $\mathcal{S}_{p}:=S_{u}\left(\omega_{1}\right) S_{u}\left(\omega-\omega_{1}\right)$, over the 2-D frequency space $\left(\omega, \omega_{1}\right)$. These two factors are represented in Fig. 8. The smallness of $S_{u}(\omega)$ over the range $\left.]\right]-\alpha ; \alpha[[$, i.e. extending almost to $\alpha$, translates into two strips where the loading spectrum $\mathcal{S}_{p}$ is negligible, see Fig. 8(b and e), and cancels out any tentative amplification that would result from the poles in the first factor. Precisely, the kernel $\mathcal{K}$ of this problem exhibits four biresonance peaks, two lower resonance peaks and several lower peaks, see Fig. 8(a). All these peaks do not influence the response, as they are located in the low-frequency range, for frequencies of order $\bar{\omega}$ actually. By virtue of the timescale separation $\bar{\omega} \ll \alpha$, all these peaks are located into the two strips where the loading spectrum is negligible. Exactly as the resonant contribution is not activated in the linear part of the response, the biresonant contribution is not activated by this kind of loading. As a result, only the peaks in $\mathcal{S}_{p}$ might remain in the product $\mathcal{K S}_{p}$. In fact, among the four peaks in the loading spectrum $\mathcal{S}_{p}$ (see Fig. 8(b and e)), the two peaks located around $\pm(\alpha, 2 \alpha)$ disappear after multiplication by the kernel $\mathcal{K}$ while the other two peaks around $( \pm \alpha, 0)$ are split into two inertial subpeaks each, as a result of the specific behavior of the kernel $\mathcal{K}$ across the plane $\omega=0$, namely two crests and one ditch as indicated in Fig. 8(d). These four peaks in the response, produced by the product of the two factors $\mathcal{K}$ and $\mathcal{S}_{p}$, are represented in Fig. 8 (c), in an anamorphose view.

A formal application of the multiple timescale spectral analysis requires a sequential focus on each peak by the use of adequate coordinate transformations. We will first focus on the peak located at $\left(\omega_{1}, \omega\right)=(\alpha, \bar{\omega})$, with the coordinate stretching $\omega_{1}=\alpha\left(1+\eta_{1}\right)$ and $\omega=\bar{\omega}\left[1+\left(\xi_{s}+\xi_{a}\right) \eta_{2}\right]$ such that

$\omega-\omega_{1}=\bar{\omega}\left[1+\left(\xi_{s}+\xi_{a}\right) \eta_{2}\right]-\alpha\left(1+\eta_{1}\right) \simeq-\alpha\left(1+\eta_{1}\right)$

because $\eta_{1}$ and $\eta_{2}$ are both of order 1 , while $\xi_{s}+\xi_{a} \ll 1$ and $\bar{\omega} \ll \alpha$. Both arguments of $H_{2}\left(\omega_{1}, \omega-\omega_{1}\right)$ are thus in the high-frequency range. Therefore, using again the approximation $\tilde{H}_{1}(\omega)=-\mathrm{i} \kappa_{m} / \omega$ in the high-frequency range, the leading order approximation of the second kernel thus reads 
a

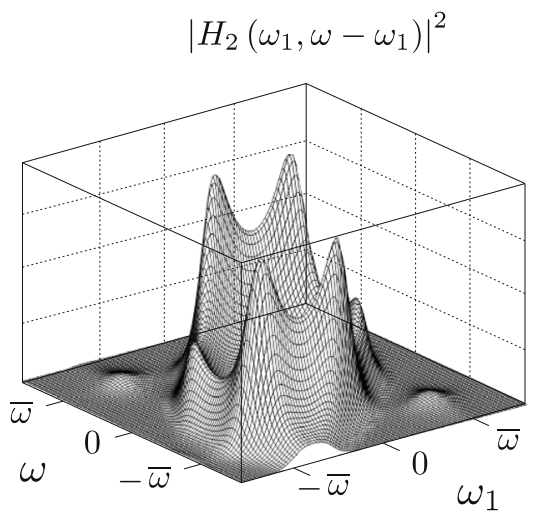

b

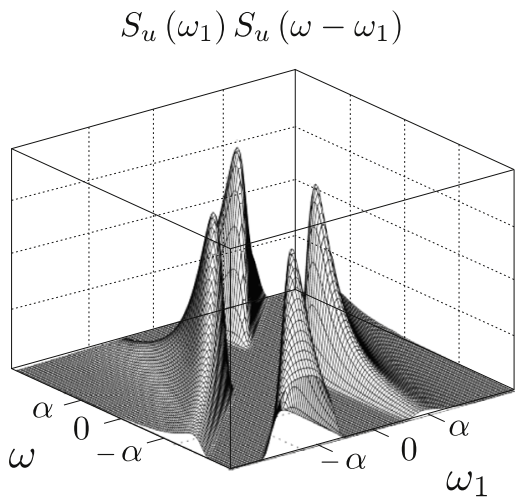

C $\left|H_{2}\left(\omega_{1}, \omega-\omega_{1}\right)\right|^{2} S_{u}\left(\omega_{1}\right) S_{u}\left(\omega-\omega_{1}\right)$

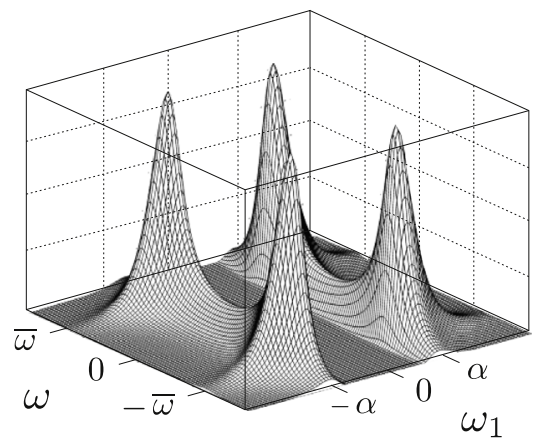

d

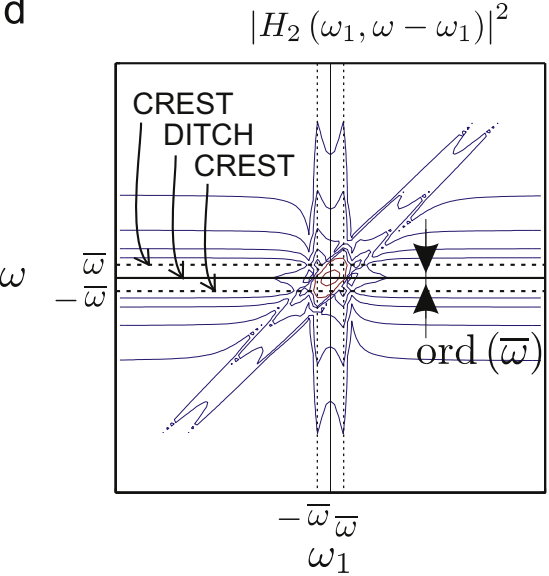

e

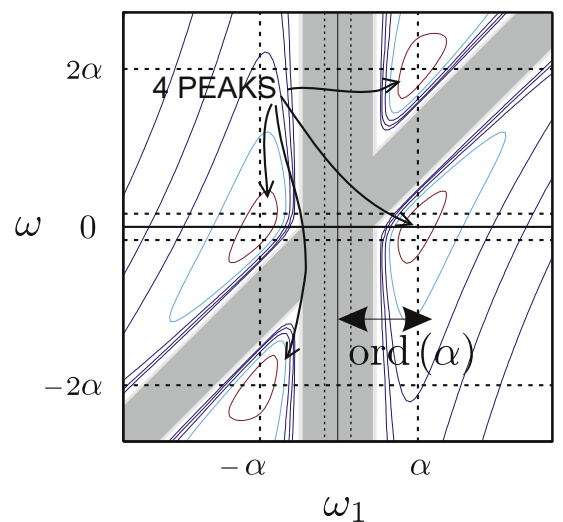

$f$

$\left|H_{2}\left(\omega_{1}, \omega-\omega_{1}\right)\right|^{2} S_{u}\left(\omega_{1}\right) S_{u}\left(\omega-\omega_{1}\right)$

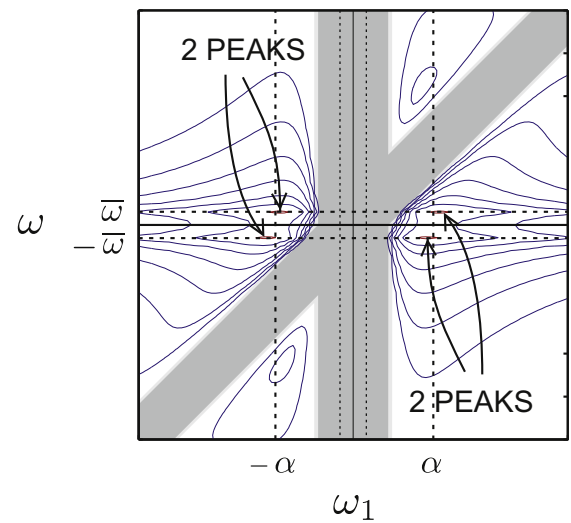

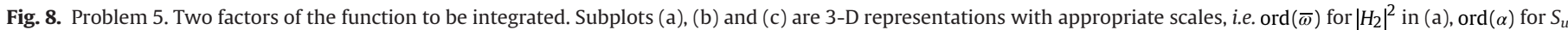
in (b) and an anamorphose view for the product in (c). To ease comparison, the corresponding contour plots (d), (e) and (f) are represented with identical axes.

$\tilde{H}_{2}\left(\omega_{1}, \omega-\omega_{1}\right)=\frac{a_{2}\left(1-\kappa_{m}\right)^{2}}{D(\omega)}$

which does not depend on $\omega_{1}$ anymore. This local behavior of $H_{2}$ features the two-crest/one-ditch pattern invoked earlier. Besides, the second factor, $S_{u}\left(\omega_{1}\right) S_{u}\left(\omega-\omega_{1}\right)$, locally behaves as

$S_{u}\left(\omega_{1}\right) S_{u}\left(\omega-\omega_{1}\right)=S_{u}\left(\alpha\left(1+\eta_{1}\right)\right) S_{u}\left(-\alpha\left(1+\eta_{1}\right)\right)=S_{u}^{2}\left(\omega_{1}\right)$.

At the opposite, it is thus a function of $\omega_{1}$ only. This study on the timescale separation has thus actually resulted in a variable separation, revealing that the frequencies $\omega$ and $\omega_{1}$ pertain, locally, to the dynamics of the system and of the loading, respectively. When considered alone, the two approximations (80) and (81) are not integrable in the far field over $\mathbb{R}^{2}$ but as a result of the variable separation, the product is seen to be bounded. When multiplied together, these two factors provide in one sweep a bounded and accurate approximation of the four peaks in the function to be integrated, those shown in Fig. 8 (c).

Leaving aside the local behavior (81) and simply substituting the $\omega_{1}$ - independent local approximation (80) into (74), the second contribution $S_{x_{2}}(\omega)$ to the power spectral density of the response reads

$\tilde{S}_{x 2}(\omega)=2 \frac{a_{2}^{2}\left(1-\kappa_{m}\right)^{4}}{|D(\omega)|^{2}} S_{u^{2}}(\omega)$

where $S_{u^{2}}(\omega)=\int_{\mathbb{R}} S_{u}\left(\omega_{1}\right) S_{u}\left(\omega-\omega_{1}\right) d \omega_{1}$ represents the power spectral density of the square of the water-particle velocity, see e.g. [40]. This function might be established in closed form for most usual wave spectral densities. Unlike the frequency content of the original process $S_{u}$, the squared water-particle velocity has a significant frequency content in the low-frequency region, as shown in Fig. 9(a).

The second integration on $\omega$, that is necessary to determine the variance $k_{2}$, finally takes the form of the mixed background-resonant contribution given by

$\int_{\mathbb{R}} \tilde{S}_{x 2}(\omega) d \omega=2 a_{2}^{2}\left(1-\kappa_{m}\right)^{4} \int_{\mathbb{R}} \frac{S_{u^{2}}(\omega)}{|D(\omega)|^{2}} d \omega$.

This second contribution to the variance of the response might thus be seen as a complete dynamic response to the quadratic component of the loading. Because $S_{u^{2}}(\omega)$ is now relatively flat and low on $[0, \bar{\omega}]$, see Fig. 9(a), the quasi-static counterpart of the response to this quadratic loading is negligible and this integral might be approximated by its sole resonant component as

$k_{2, b r}=2 a_{2}^{2}\left(1-\kappa_{m}\right)^{4} S_{u^{2}}(\bar{\omega}) \frac{\pi \bar{\omega}}{2\left(\xi_{s}+\xi_{a}\right)}$.

Summing up the first-order background and second-order mixed background/resonant components of the response, one finally gets

$k_{2}=k_{2, b}+k_{2, b r}=\kappa_{m}^{2} k_{2, \int u}+a_{2}^{2}\left(1-\kappa_{m}\right)^{4} S_{u^{2}}(\bar{\omega}) \frac{\pi \bar{\omega}}{\xi_{s}+\xi_{a}}$

where the order of integration has dropped by 2 in this case since no biresonance peaks are present in the response. To illustrate the quality of the approximation, Fig. 9(b) represents, in a very good agreement, the exact power spectral density $S_{x}(x)$ from (73), the two approximations $\tilde{S}_{x_{1}}(\omega)$ and $\tilde{S}_{x_{2}}(\omega)$ that led to the final approximation (85), as well as their sum $\tilde{S}_{x}(x)$. The numerical values considered for the illustration are taken from [34]. 
a

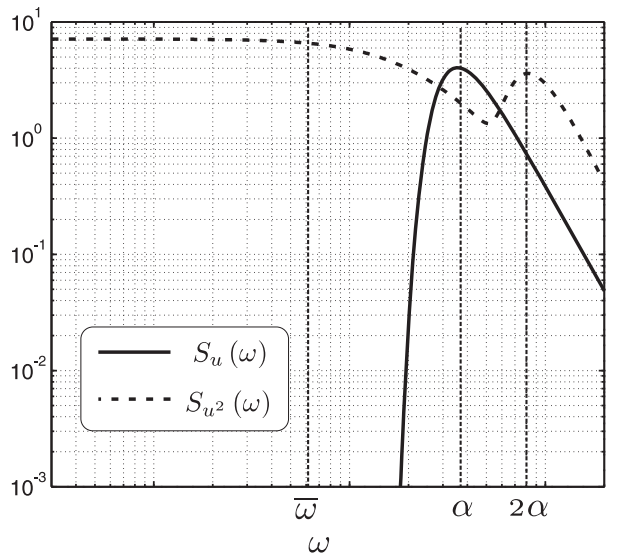

b

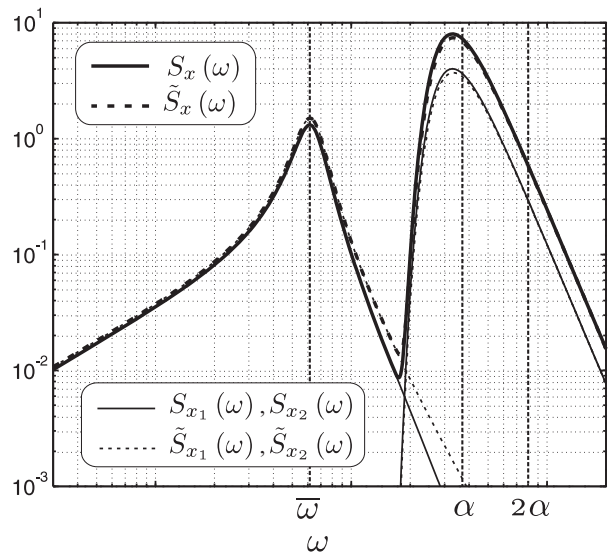

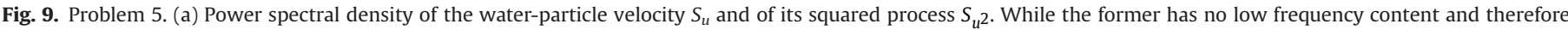

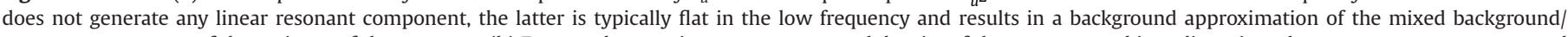

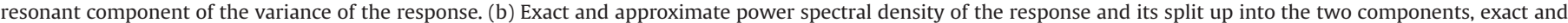
approximate, too. The background component is in the high frequency range $\alpha$ while the mixed component is in the low frequency range $\bar{\omega}$.

\section{Conclusions}

The multiple timescale spectral analysis is a formal extension of the well-known background/resonant decomposition to problems involving MDOF systems, nonlinearity, non-Gaussianity or even non-stationarity. It deals with the statistical cumulants of the response as well as its time-derivatives or any other information related to the spectral description. Following the general guidelines of the method, that were illustrated with various examples, the timescale separation between the loading and the structural properties results in a reduction of the dimensionality of the integrals that need to be computed in spectral analyses. This in turn translates into massive computational savings. The key ingredients of the method are (i) the analytical expression of the structural kernel (at least along the direction that is independent of the loading, in the neighborhood of a local contribution) and (ii) the need for a local estimation of the loading only, which thus prevents from computing the properties of the loading across the whole frequency space.

Collaborative ongoing works by the author indicates that the method may also be applied to efficient stochastic linearization and evolutionary problems.

\section{References}

[1] J.S. Bendat, A.G. Piersol, Engineering Applications of Correlation and Spectral Analysis, 2nd ed., John Wiley and Sons, Hoboken, New Jersey, 1993.

[2] M. Grigoriu, Stochastic Calculus: Applications in Science and Engineering, Springer-Verlag, Birkhauser, 2002.

[3] A. Skorokhod, F. Hoppensteadt, H. Salehi, Random Perturbation Methods with Applications in Science and Engineering, Springer, Springer Verlag - New-York, Inc., 2002.

[4] G.M. Jenkins, D.G. Watts, Spectral Analysis and Its Applications, Holden-Day, San Francisco, California, 1998.

[5] A. Preumont, Random Vibration and Spectral Analysis, Kluwer Academic Publishers, Lausanne, 1994.

[6] R.W. Clough, J. Penzien, Dynamics of Structures, 2nd ed., McGraw-Hill, NewYork, 1993.

[7] Y.K. Lin, G.Q. Cai, Probabilistic Structural Dynamics: Advanced Theory and Applications, McGraw-Hill, New York, 2004.

[8] Y. Goda, Random Seas and Design of Maritime Structures, World Scientific Danvers, Massachussets, 2000.

[9] C.P. Robert, G. Casella, Monte Carlo Statistical Methods, 2nd ed., 2004.

[10] G.I. Schuëller, A state-of-the-art report on computational stochastic mechanics, Probab. Eng. Mech. 12 (4) (1997) 197-321.

[11] S. Benfratello, G. Falsone, Non-gaussian approach for stochastic-analysis of offshore structures, J. Eng. Mech.-Asce 121 (11) (1995) 1173-1180.
[12] G.Q. Cai, Y. Suzuki, Response of systems under non-Gaussian random excitations, Nonlinear Dyn. 45 (1-2) (2006) 95-108.

[13] K.R. Gurley, M.A. Tognarelli, A. Kareem, Analysis and simulation tools for wind engineering, Probab. Eng. Mech. 12 (1) (1997) 9-31.

[14] L.D. Lutes, S.L.J. Hu, Nonnormal stochastic response of linear-systems, J. Eng. Mech.-Asce 112 (2) (1986) 127-141.

[15] G. Muscolino, Linear systems excited by polynomial forms of non-Gaussian filtered processes, Probab. Eng. Mech. 10 (1) (1995) 35-44.

[16] A. Papoulis, Probability, Random Variables, and Stochastic Processes, McGraw Hill, New York, 1965.

[17] M.B. Priestley, Evolutionary spectra and non-stationary processes, J. R. Stat. Soc. Ser. B (Methodol.) 27 (2) (1965) 204-237.

[18] I.A. Kougioumtzoglou, Pol D. Spanos, Nonlinear mdof system stochastic response determination via a dimension reduction approach, Comput. Struct. 126 (0) (2013) 135-148.

[19] A. der Kiureghian, A. Neuenhofer, Response spectrum method for multi-support seismic excitations, Earthq. Eng. Struct. Dyn. 21 (8) (1992) 713-740.

[20] R.S. Harichandran, A. Hawwari, B.N. Sweidan, Response of long-span bridges to spatially varying ground motion a, J. Struct. Eng. 122 (5) (1996) 476-484

[21] J.B. Roberts, PolD. Spanos, Random Vibration and Statistical Linearization, Dover Publications, New York, 1999.

[22] M.G. Donley, Pol D. Spanos, Dynamic Analysis of Non-linear Structures by the Method of Statistical Quadratization, Springer-Verlag, New York, 1990.

[23] P.D. Spanos, G. Failla, M. Di Paola, Spectral approach to equivalent statistical quadratization and cubicization methods for nonlinear oscillators, J. Eng. Mech.-Asce 129 (1) (2003) 31-42.

[24] A. Kareem, J. Zhao, M.A. Tognarelli, Surge response statistics of tension leg platforms under wind and wave loads: a statistical quadratization approach, Probab. Eng. Mech. 10 (4) (1995) 225-240.

[25] K. Worden, G. Manson, Random vibrations of a multi-degree-of-freedom nonlinear system using the Volterra series, J. Sound Vib. 226 (2) (1999) 397-405.

[26] L. Carassale, A. Kareem, Dynamic analysis of complex systems by Volterra approach, Comput. Stoch. Mech. (2003), 107-117.

[27] A. Naess, O. Gaidai, The asymptotic behaviour of second-order stochastic Volterra series models of slow drift response, Probab. Eng. Mech. 22 (4) (2007) 343-352.

[28] A. Zerva, Response of multi-span beams to spatially incoherent seismic ground motions, Earthq. Eng. Struct. Dyn. 19 (6) (1990) 819-832.

[29] C. de Boor, On Writing an Automatic Integration Algorithm, Academic Press, New York, 1971, pages pp. 201-209.

[30] A. Quarteroni, R. Sacco, F. Saleri, Numerical Mathematics, volume 1 of Texts in Applied Mathematics, 2nd ed., Springer-Verlag, Berlin, 2007.

[31] V. Denoël, Application of stochastic analysis methods to the study of the effects of wind on civil engineering structures (Ph.D. thesis), 2005.

[32] P. van Dooren, L. de Ridder, An adaptive algorithm for numerical integration over an n-dimensional cube, J. Comput. Appl. Math. 2 (3) (1976) 207-217.

[33] S. Benfratello, M. Di Paola, P.D. Spanos, Stochastic response of mdof wind excited structures by means of Volterra series approach, J. Wind Eng. Ind Aerodyn. 74-6 (1998) 1135-1145.

[34] L. Carassale, A. Kareem, Modeling nonlinear systems by Volterra series, J. Eng. Mech.-ASCE 136 (2010) 801-818.

[35] F. Ma, Extension of second moment analysis to vector-valued and matrix-valued functions, Int. J. Non-Linear Mech. 22 (3) (1987) 251-260.

[36] C. Papadimitriou, L.D. Lutes, Stochastic cumulant analysis of mdof systems with polynomial type nonlinearities, Probab. Eng. Mech. 11 (1) (1996) 1-13.

[37] V. Gusella, A.L. Materazzi, Non-Gaussian along-wind response analysis in time and frequency domains, Eng. Struct. 22 (1) (2000) 49-57. 
[38] A.G. Davenport, The application of statistical concepts to the wind loading of structures, in: Proceedings of the Institute of Civil Engineers, vol. 19, 1961, pp. 449-472.

[39] R.L. Stratonovich, Topics in the Theory of Random Noise, vol. 1., Science Publishers, Inc., New York, 1963.

[40] A. Kareem, Nonlinear wind velocity term and response of compliant offshore structures, J. Eng. Mech. 110 (October (10)) (1984) 1573-1578.

[41] S.H. Crandall, Perturbation techniques for random vibration of nonlinear systems, J. Acoust. Soc. Am. 35 (11) (1963) 1700-1705.

[42] V. Denoël, Estimation of modal correlation coefficients from background and resonant responses, Struct. Eng. Mech.: Int. J. 32 (6) (2009) 725-740.

[43] V. Denoël, On the background and biresonant components of the random response of single degree-of-freedom systems under non-Gaussian random loading, Eng. Struct. 33 (8) (2011) 2271-2283.

[44] H.K. Khalil, Nonlinear Systems, Engelwood Cliffs, New Jersey, 2002.

[45] E. Bedrosian, S.O. Rice, Output properties of Volterra systems (nonlinear systems with memory) driven by harmonic and gaussian inputs, Proc. IEEE 59 (12) (1971) 1688-1707.

[46] M. Schetzen, The Volterra and Wiener Theories of Nonlinear Systems, 1980

[47] A. Swami, G.B. Giannakis, G. Zhou, Bibliography on higher-order statistics, Signal Process. 60 (1) (1997) 65-126.

[48] M. Grigoriu, Response of offshore structures to random waves, J. Eng. Mech.Asce 113 (8) (1986) 729-744.

[49] J. Kevorkian, J.D. Cole, Multiple Scale and Singular Perturbation Methods. Applied Mathematical Sciences, Springer-Verlag, Berlin, 1996.
[50] G. Wentzel, Eine verallgemeinerung der quantenbedingungen für die zwecke der wellenmechanik, Z. Phys. (38) (1926) 518-529.

[51] H.A. Kramers, Wellenmechanik und halbzahlige quantisierung, Z. Phys. 39 (1926) 828-840.

[52] Léon Brillouin, La mécanique ondulatoire de schrödinger; une méthode générale de résolution par approximations successives, C. R. Acad. Sci. 183 (1926) 24-26.

[53] E.J. Hinch, Perturbation Methods, vol. 1, Cambridge University Press, Cambridge, 1991.

[54] V. Denoël, L. Carassale, Perturbation Methods for the Determination of the Statistical Response of Nonlinear Volterra Systems, 2014.

[55] C.M. Bender, S.A. Orszag, Advanced Mathematical Methods for Scientists and Engineers. New Ed., 1999.

[56] C. Dyrbye, S.O. Hansen, Wind Loads on Structures, John Wiley and Sons, Chichester, England, 1997.

[57] E. Simiu, R. Scanlan, Wind Effects On Structures, 3rd ed., John Wiley and Sons, New York, 1996.

[58] M. Gu, X.-Y. Zhou, An approximation method for resonant response with coupling modes of structures under wind action, J. Wind Eng. Ind. Aerodyn. 97 (11-12) (2009) 573-580.

[59] S.K. Chakrabarti, Nonlinear Methods in Offshore Engineering, Elsevier, Amsterdam, 1990.

[60] A. Kareem, M.A. Tognarelli, K.R. Gurley, Modeling and analysis of quadratic term in the wind effects on structures, J. Wind Eng. Ind. Aerodyn. 74 (6) (1998) 1101-1110. 